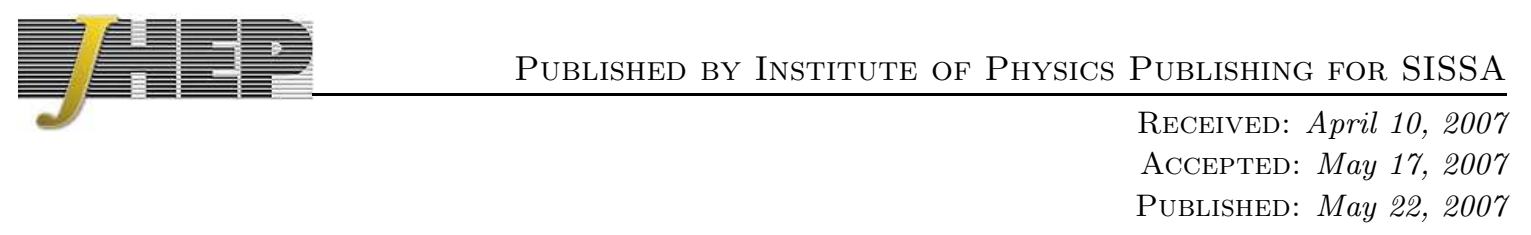

\title{
Multi-trace deformations in AdS/CFT: exploring the vacuum structure of the deformed CFT
}

\author{
loannis Papadimitriou \\ DESY Theory Group, \\ Notkestrasse 85, D-22603 Hamburg, Germany, and \\ Center for Mathematical Physics, \\ Bundesstrasse 55, D-20146 Hamburg, Germany \\ E-mail: ioannis.papadimitriou@desy.de
}

\begin{abstract}
We present a general and systematic treatment of multi-trace deformations in the AdS/CFT correspondence in the large $\mathrm{N}$ limit, pointing out and clarifying subtleties relating to the formulation of the boundary value problem on a conformal boundary. We then apply this method to study multi-trace deformations in the presence of a scalar VEV, which requires the coupling to gravity to be taken into account. We show that supergravity solutions subject to 'mixed' boundary conditions are in one-to-one correspondence with critical points of the holographic effective action of the dual theory in the presence of a multi-trace deformation, and we find a number of new exact analytic solutions involving a minimally or conformally coupled scalar field satisfying 'mixed' boundary conditions. These include the generalization to any dimension of the instanton solution recently found in hep-th/0611315. Finally, we provide a systematic method for computing the holographic effective action in the presence of a multi-trace deformation in a derivative expansion away from the conformal vacuum using Hamilton-Jacobi theory. Requiring that this effective action exists and is bounded from below reproduces recent results on the stability of the AdS vacuum in the presence of 'mixed' boundary conditions.
\end{abstract}

KEYWORDS: Gauge-gravity correspondence, AdS-CFT Correspondence. 


\section{Contents}

1. Introduction and summary of results 1

2. Multi-trace deformations in QFTs with a large $N$ limit 3

3. The boundary value problem for the Klein-Gordon operator in AlAdS spaces

3.1 The variational problem in the presence of a conformal boundary

3.2 Boundary conditions 10

3.3 Solution of the boundary value problem 11

3.4 The on-shell action and the AdS/CFT dictionary 12

4. Toy model 14

4.1 General solution with linear boundary conditions 14

4.2 Vacua with non-linear boundary conditions 16

5. Effective action from Hamilton's characteristic function 18

6. Minimal coupling 22

6.1 Two-derivative effective action for conformal boundary conditions 23

6.2 Minisuperspace approximation 28

6.3 The ' $2 / 3$ ' potential 30

7. Conformal coupling 33

7.1 Two-derivative effective action for conformal boundary conditions

7.2 Minisuperspace approximation 36

7.3 Instantons

A. The variational problem and Hamilton-Jacobi equations 45

\section{Introduction and summary of results}

Multi-trace deformations have been studied extensively in the context of the AdS/CFT correspondence in the large $N$ limit, both classically [1-7] and at the one-loop level [810]. Most of this work, however, has focused on the effect of multi-trace deformations on the conformal vacuum, in which case the back-reaction to the geometry can be ignored. If the deforming operator though is allowed to acquire a non-zero VEV, then the back-reaction can no longer be ignored and the coupling to gravity must be taken into account. Only 
recently have multi-trace deformations in the presence of a scalar VEV been considered, mainly in the context of Designer Gravity [11-21].

In order for a CFT to admit multi-trace deformations it must contain operators with low enough dimension. For double- or higher-trace deformations built out of a single operator, for example, not to be irrelevant, the operator must have conformal dimension $\Delta \leq d / 2$ in $d$ dimensions. For scalar operators, for example, this means that the operator must have the 'non-standard' $\Delta_{-}$dimension. This constraint, together with unitarity, which imposes a lower bound on the dimension $\Delta$, severely restricts the CFTs admitting multi-trace deformations. The possibilities are further narrowed if one insists that the undeformed CFT be supersymmetric. Since the AdS/CFT dictionary relates multi-trace deformations in the large $N$ limit to a choice of boundary conditions for the dual bulk supergravity fields [1], these restrictions on the conformal dimension of the operator translate into a condition on the mass of the dual supergravity fields for them to admit the necessary generalized boundary conditions. We are then interested in gauged supergravities that admit AdS vacua and have fields with mass close to the Breitenlohner-Freedman bound [22].

Both the maximal gauged supergravities in four and five dimensions contain scalars with the right mass, and indeed black hole solutions with scalar hair that satisfy generalized boundary conditions were found numerically in 12], following earlier work in three dimensions [11. Smooth instantons and gravitational soliton solutions of $\mathcal{N}=8 D=4$ gauged supergravity with generalized boundary conditions were also found numerically in [13], and shown to be related to a Big Crunch geometry. More recently, exact solutions of $\mathcal{N}=8 D=4$ gauged supergravity obeying generalized boundary conditions were found analytically in [23] and [24] and uplifted to eleven dimensions. The AdS/CFT identifies these solutions with 'vacua' or 'states' in the dual deformed CFT. In particular, the extrema of the large $N$ quantum effective action for the VEV of the deforming operator are in one-to-one correspondence with bulk solutions satisfying the relevant boundary conditions. These bulk solutions then provide a window into the vacuum structure of the deformed theory.

A very interesting question, in particular, is whether the conformal vacuum - which generically remains a vacuum of the deformed theory - is stable or not under certain boundary conditions. The instantons found in [12] and [24] show that it is not, under the particular AdS-invariant boundary conditions that these instantons satisfy, since these mediate the tunneling of the conformal vacuum to an instability region. This, of course, does not contradict any of the well known stability theorems [25-27], because these apply only to certain special boundary conditions. The question of stability with more general boundary conditions corresponding to multi-trace deformations has been addressed recently in the context of Designer Gravity [18-21. The approach followed is a generalization of the spinorial argument of [28], but as in the earlier work [26, 27] no supersymmetry is required. The argument only relies on the existence 'fake Killing spinors', which themselves can be constructed from a 'fake superpotential'. Non-perturbative stability then follows from the existence of a suitable 'fake superpotential'.

However, the AdS/CFT correspondence allows us to address the problem of nonperturbative stability from a completely different point of view. Namely, if we knew the 
effective action of the dual theory, then we would be able to address the question of stability/instability directly. We will show that the effective action can be computed holographically in a derivative expansion using Hamilton-Jacobi theory [29]. Requiring that this effective action exists and it is stable reproduces all known stability results, including the recent results in the case of generalized boundary conditions. This agreement can be traced to the fact that both arguments require global existence of a suitable 'fake superpotential'. In the latter case, however, this is interpreted as Hamilton's characteristic function, which allows us to immediately generalize these results to other systems, such as conformally coupled scalars.

The paper then is organized as follows. In section 2 we review a general description of multi-trace deformations in the large $N$ limit, which relies on large $N$ factorization. This will make manifest the correspondence between multi-trace deformations on the boundary and boundary conditions in the bulk in section 3, where we revisit the boundary value problem and the possible boundary conditions for the Klein-Gordon operator in asymptotically locally AdS spaces. In particular, we present a general systematic method to address multi-trace deformations and to properly account for the fact that the boundary is a conformal boundary - as opposed to a hard boundary. As we show, this automatically removes the divergences associated with the infinite volume of the space. Although we present these results for scalar fields, they immediately generalize to any field admitting boundary conditions corresponding to multi-trace deformations. In section 1 then we demonstrate the general method in the case of a free massive scalar field in a fixed AdS background, reproducing in a concise way a number of known results. We then move on in section 5 to include gravity and we describe in detail our method for computing the holographic effective action of the dual theory in a systematic way based on Hamilton-Jacobi theory. This method is then applied to the cases of scalars minimally and conformally coupled to gravity in sections 6 and 0 respectively, which contain our main results. In section 6 we generalize the non-supersymmetric Poincaré domain wall solutions found in 23] to arbitrary dimension, while the same is done for the instanton solution found in 24] in section 7. Moreover, we find all possible domain wall solutions - both flat and curved for the conformally coupled scalar in any dimension, and we show that this completely determines the two-derivative effective action of the dual theory. Some technical results regarding the variational problem for minimally and conformally coupled scalars, as well as the Hamilton-Jacobi method for these systems, are collected in the appendix.

\section{Multi-trace deformations in QFTs with a large $N$ limit}

In a quantum field theory with a standard large $N$ limit, large $N$ factorization allows for a universal description of generic multi-trace deformations. As we now briefly review, the effect of such a deformation can most naturally be described in terms of the generating functional of the deforming operator and its Legendre transform [7].

Let $\mathcal{O}(x)$ be a local, generically composite, gauge-invariant and single-trace operator transforming in some representation of the relevant rank $N$ group. For concreteness we take this to be the adjoint representation and we normalize the operator such that $\langle\mathcal{O}\rangle=O\left(N^{0}\right)$ 
as $N \rightarrow \infty$. The dynamics of $\mathcal{O}(x)$ is encoded in the generating functional of connected correlators, $W[J]$, which can be represented as a path integral over the fundamental degrees of freedom, $\{\phi\}$, of the theory, weighted by the action $S[\phi]$, as

$$
e^{-W[J]}=\int[d \phi] e^{-S[\phi]-N^{2} \int d^{d} x J(x) \mathcal{O}(x)} .
$$

Since $W[J]$ is $O\left(N^{2}\right)$ as $N \rightarrow \infty$, it is convenient to work instead with $w[J] \equiv N^{-2} W[J]$. In particular, the one-point function of $\mathcal{O}(x)$ in the presence of a source is given by

$$
\sigma(x) \equiv\langle\mathcal{O}\rangle_{J}=\frac{\delta w[J]}{\delta J} .
$$

Alternatively, the dynamics can be encoded in the Legendre transform of the generating functional, $\Gamma[\sigma]$, given by

$$
e^{-\Gamma[\sigma]}=\int[d J] e^{-N^{2} w[J]+N^{2} \int d^{d} x J(x) \sigma(x)} .
$$

$\Gamma[\sigma]$ is known as the effective action of the local operator $\mathcal{O}(x)$, or the generating functional of 1PI diagrams. Again, it is useful to introduce the $O\left(N^{0}\right)$ quantity $\bar{\Gamma}[\sigma]=N^{-2} \Gamma[\sigma]$, such that

$$
J(x)=-\frac{\delta \bar{\Gamma}[\sigma]}{\delta \sigma} .
$$

Suppose now that the action is deformed by a function, $f(\mathcal{O})$, of the local operator $\mathcal{O}(x)$ as $S_{f}[\phi]=S[\phi]+N^{2} \int d^{d} x f(\mathcal{O})$. In the following we will only consider deformations for which $f(0)=0$. The question we want to address now is how this deformation modifies the functionals $w[J]$ and $\bar{\Gamma}[\sigma]$. As we now show, large $N$ factorization allows for a very simple and universal answer in the large- $N$ limit, which is summarized in table 1. Of course, beyond the large $N$ approximation, the answer to this question is non-universal and much more involved, since the operator $\mathcal{O}(x)$ will generically mix with other operators at $1 / N$ order. We will only consider the leading large $N$ behavior here.

Consider first the generating functional in the deformed theory, which is given by

$$
\begin{aligned}
& e^{-N^{2} w_{f}\left[J_{f}\right]}=\int[d \phi] e^{-S[\phi]-N^{2} \int d^{d} x\left(J_{f} \mathcal{O}+f(\mathcal{O})\right)} \\
& =\int[d \phi] e^{-S[\phi]-N^{2} \int d^{d} x\left(J \mathcal{O}+f(\mathcal{O})-f^{\prime}(\sigma) \mathcal{O}\right)} \\
& \stackrel{N \rightarrow \infty}{\approx} e^{-N^{2} w[J]} e^{-N^{2} \int d^{d} x\left(f(\sigma)-\sigma f^{\prime}(\sigma)\right)},
\end{aligned}
$$

where we introduced $J \equiv J_{f}+f^{\prime}(\sigma)$ in the second line in order to remove the linear term from $f(\mathcal{O})$ so that large $N$ factorization can be used in the last step. This proves the result shown in the third row of table 1. Similarly, the effective action in the deformed theory is given by

$$
\begin{aligned}
e^{-N^{2} \bar{\Gamma}_{f}[\sigma]} & =\int\left[d J_{f}\right] e^{-N^{2} w_{f}\left[J_{f}\right]+N^{2} \int d^{d} x J_{f} \sigma} \\
& { } \rightarrow \infty \int[d J] e^{-N^{2} w[J]} e^{-N^{2} \int d^{d} x\left(f(\sigma)-\sigma f^{\prime}(\sigma)\right)} e^{N^{2} \int d^{d} x\left(J-f^{\prime}(\sigma)\right) \sigma} \\
& =e^{-N^{2} \bar{\Gamma}[\sigma]-N^{2} \int d^{d} x f(\sigma)},
\end{aligned}
$$




\begin{tabular}{|c|c|c|}
\hline & Undeformed & Deformed \\
\hline Source & $J$ & $J_{f}=J-f^{\prime}(\sigma)$ \\
VEV & $\sigma$ & $\sigma_{f}=\sigma$ \\
Generating functional & $w[J]$ & $w_{f}\left[J_{f}\right]=w[J]+\left.\int d^{d} x\left(f(\sigma)-\sigma f^{\prime}(\sigma)\right)\right|_{\sigma=\delta w / \delta J}$ \\
Effective action & $\bar{\Gamma}[\sigma]$ & $\bar{\Gamma}_{f}[\sigma]=\bar{\Gamma}[\sigma]+\int d^{d} x f(\sigma)$ \\
\hline
\end{tabular}

Table 1: The effect of a generic multi-trace deformation on the generating functional and on the effective action in the large $N$ limit.

where we have used $\left[d J_{f}\right]=[d J]$. This justifies the entry in the last row of table 1. As we will review below, these universal results make manifest the fact that the AdS/CFT dictionary maps multi-trace deformations of the boundary theory to a modification of the boundary conditions imposed on the bulk fields. Before, however, we need to understand the boundary value problem for such bulk fields in AdS.

\section{The boundary value problem for the Klein-Gordon operator in AlAdS spaces}

The gauge/gravity duality generically relates a theory of gravity in an asymptotically locally anti de Sitter (AlAdS) space $\mathcal{M}$ (see [30] for a definition of an AlAdS space in the context of the gauge/gravity duality) to a non-gravitational theory residing on the conformal boundary $\partial \mathcal{M}$ of $\mathcal{M}$. Multi-trace deformations of the boundary theory are then related to the choice of boundary conditions imposed on the bulk fields [1 - 5, 8, 6, 7]. The fact that the boundary of an AlAdS space is a conformal boundary, however, demands some extra care when analyzing the boundary value problem. In particular, any rigorous treatment should account for the following fact [31]:

By the very definition of a 'conformal boundary', any bulk field does not induce a field on the boundary, but rather a field up to Weyl rescalings, i.e. a 'conformal class'. It follows that, in the absence of a conformal anomaly, any boundary condition must be imposed on the conformal class and not on a conformal representative. In other words, any boundary condition must be imposed on a 'class function'. Although, this cannot be achieved if a conformal anomaly is present, in that case one must ensure that the boundary condition is imposed on a quantity that has a well defined transformation under Weyl rescalings.

This requirement, which we will make more precise and concrete below, has a number of important and inevitable consequences that are often overlooked:

- The well known boundary covariant counterterms must be added to the action before one can study the variational problem and impose boundary conditions. 
- With the standard Dirichlet boundary conditions, the one-point function of an operator $\mathcal{O}(x)$ is in general not given by the normalizable mode of the corresponding bulk field. It is given by the renormalized radial momentum [32]. In general, the two differ by a local functional of the non-normalizable mode, which is necessary to ensure that the Ward identities are fulfilled [33, 30]. In particular, it is the relation between the non-normalizable mode and the renormalized radial momentum which is fundamentally related to the choice of boundary conditions and not the relation between the non-normalizable and normalizable modes. Only when the two happen to agree is one justified to use the normalizable mode instead of the renormalized momentum.

In view of these subtleties, and for the sake of completeness, we find it worthwhile to devote this section to a careful and systematic analysis of the boundary value problem and to review how the AdS/CFT dictionary relates the choice of boundary conditions for the bulk fields to multi-trace deformations of the boundary theory. We also take this opportunity to spell out the formalism and notation which will be used in the subsequent sections.

The metric on an AlAdS manifold, $\mathcal{M}$ takes the form ${ }^{1}$

$$
d s^{2}=d r^{2}+\gamma_{i j}(r, x) d x^{i} d x^{j},
$$

where $\gamma_{i j}(r, x) \sim e^{2 r / l} g_{(0) i j}(x)$ as $r \rightarrow \infty$ and hence, the conformal boundary, $\partial \mathcal{M}$, is located at $r=\infty$. The metric $g_{(0) i j}(x)$ is a metric on the conformal boundary, or more precisely, a representative of the conformal class of boundary metrics. AlAdS metrics arise naturally as solutions of Einstein's equations with a negative cosmological constant, possibly including matter whose stress tensor falls fast enough asymptotically [30].

To set up the formalism, we will study the simplest possible boundary value problem on the background of such a manifold, namely that of the Klein-Gordon equation for a scalar field,

$$
\left(-\square_{g}+m^{2}\right) \phi=0
$$

One can include interactions in this equation, and we will do so later on, but these are irrelevant for the boundary value problem as long as they do not modify the asymptotic form of the metric. Any solution of (3.2) in the background (3.1) takes the asymptotic form

$$
\phi \sim\left\{\begin{array}{l}
e^{-\Delta_{-} r / l}\left(\phi_{-}(x)+\cdots\right)+e^{-\Delta_{+} r / l}\left(\phi_{+}(x)+\cdots\right), m^{2} l^{2}>-(d / 2)^{2}, \\
e^{-d r / 2 l} \frac{r}{l}\left(\phi_{-}(x)+\cdots\right)+e^{-d r / 2 l}\left(\phi_{+}(x)+\cdots\right), m^{2} l^{2}=-(d / 2)^{2},
\end{array}\right.
$$

where $\Delta_{ \pm}, \Delta_{+} \geq \Delta_{-}$, are the roots of the equation $m^{2} l^{2}=\Delta(\Delta-d)$, and the functions $\phi_{-}(x)$ and $\phi_{+}(x)$, known respectively as the non-normalizable and normalizable modes, are totally arbitrary functions of the transverse coordinates, $x^{i}$. A boundary condition amounts to a choice of a function $J\left(\phi_{-}, \phi_{+}\right)$of the two modes that is kept constant on the boundary, thus reducing by half the degrees of freedom. The best known example is

\footnotetext{
${ }^{1}$ We use Euclidean signature throughout this paper.
} 
that of Dirichlet boundary conditions, where one chooses $J\left(\phi_{-}, \phi_{+}\right)=\phi_{-}$, and so the only degree of freedom remaining is the normalizable mode $\phi_{+}$.

We could now try to study boundary conditions by considering different choices of the function $J\left(\phi_{-}, \phi_{+}\right)$, which is in fact what has been done in the vast majority of the literature on the subject. Although, this approach happens to work in certain cases, generically it is fundamentally problematic for two closely related reasons. Firstly, the quantity $\phi_{+}$, contrary to the non-normalizable mode $\phi_{-}$, has no well defined transformation under Weyl rescalings and hence it is an ill defined quantity from the boundary point of view. Secondly, precisely because $\phi_{+}$is not well defined on the boundary, the function $J\left(\phi_{-}, \phi_{+}\right)$one would use to define the boundary condition does not have a direct meaning (as a source) on the boundary. As we mentioned above and we will now explain in detail, both problems are resolved if one replaces $\phi_{+}$in this analysis with the renormalized radial momentum [32], which does have a definite transformation under Weyl rescalings and hence it is a well defined boundary quantity, like $\phi_{-}$. The renormalized radial momentum in general differs from $\phi_{+}$by a local functional of $\phi_{-}$, which is essential to ensure that the Ward identities are satisfied [30]. It is precisely these local terms that make the renormalized momentum have a well defined transformation rule under Weyl rescalings. Unless these local terms happen to vanish, and one needs to demonstrate that they do, we are forced to use the radial Hamiltonian formulation in order to discuss generalized boundary conditions consistently.

\subsection{The variational problem in the presence of a conformal boundary}

Since the conformal boundary, $\partial \mathcal{M}$, is located at infinity, we need to introduce a regulating surface, $\Sigma_{r}$, diffeomorphic to the boundary, but at a finite value of the radial coordinate $r$. One then formulates the variational problem on $\Sigma_{r}$ and in the end the regulator is removed by sending $r \rightarrow \infty$. It is crucial, however, to keep in mind that the conformal boundary $\partial \mathcal{M}$ and the hard boundary introduced by the regulating surface $\Sigma_{r}$ are very different in nature. In particular, the regulating surface breaks explicitly the invariance under Weyl rescalings that the conformal boundary possesses. It follows that not any variational problem that makes sense on $\Sigma_{r}$ will make sense as the regulator is removed. It will only make sense provided the variational problem on $\Sigma_{r}$ is formulated in terms of conformal class functions. Before we discuss how this can be achieved, though, let us consider the general variational problem on the regulating surface $\Sigma_{r}$.

Given an action $S[\phi]$ on a space $\mathcal{M}_{r}$ with a boundary $\Sigma_{r}=\partial \mathcal{M}_{r}$, one is naturally led to the radial Hamiltonian formulation of the bulk dynamics by considering the variational problem for the action $S[\phi]$. Indeed, a generic variation of the bulk action with respect to the scalar field generates a boundary term of the form ${ }^{2}$

$$
\delta_{\phi} S=\int_{\Sigma_{r}} d^{d} x \pi_{\phi} \delta \phi
$$

where $\pi_{\phi}$ is the canonical momentum conjugate to $\phi$ and the Hamiltonian 'time' is taken to be the radial coordinate $r$ orthogonal to the boundary $\Sigma_{r}$. For the bulk action giving

\footnotetext{
${ }^{2}$ The complete expressions for the variation of the action when the scalar is minimally or conformally coupled to gravity are presented in appendix A.
} 
the Klein-Gordon equation as the equation of motion, the canonical momentum is simply $\pi_{\phi}=\sqrt{\gamma} \dot{\phi}$, where the dot denotes a derivative with respect to the radial coordinate, $r$. Were $\Sigma_{r}$ the true boundary, we could impose any boundary condition compatible with the variation (3.4). But we actually have to send $r \rightarrow \infty$ in the end, and the integrand in (3.4) does not have a well defined transformation under shifts in $r$. Hence, if we impose a boundary condition on (3.4), we will not be able to 'push' this boundary condition to the true boundary at $r \rightarrow \infty$. What we need to do first, is to find a covariant way of modifying (3.4), without changing the bulk dynamics of course, such that the result has a well defined transformation - in fact, remains invariant - under radial shifts. A systematic way of constructing quantities which are both covariant with respect to $\Sigma_{r}$ diffeomorphisms and have a well defined transformation under radial shifts in the vicinity of the conformal boundary $\partial \mathcal{M}$, is based on the following observation [32]: The asymptotic form $\phi \sim e^{-\Delta_{-} r / l} \phi_{-}(x)$ of the scalar field allows us to write the radial derivative in the from $^{3}$

$$
\partial_{r}=\int_{\Sigma_{r}} d^{d} x \dot{\phi} \frac{\delta}{\delta \phi} \sim \frac{1}{l} \delta_{D}
$$

where

$$
\delta_{D}=-\Delta_{-} \int_{\Sigma_{r}} d^{d} x \phi \frac{\delta}{\delta \phi}
$$

is the dilatation operator, and $\sim$ means that only the leading asymptotic behavior as $r \rightarrow \infty$ is shown. It follows that quantities that have a well defined transformation under radial shifts correspond to eigenfunctions of the dilatation operator. However, by trading the radial derivative for the dilatation operator we also automatically achieve covariance with respect to $\Sigma_{r}$ diffeomorphisms. The dilatation operator (3.6), therefore, provides us with a way to decompose the integrand in (3.4), which does not transform in a controlled way under radial translations, into pieces with a well defined transformation.

This is achieved by expanding the canonical momentum $\pi_{\phi}$ in eigenfunctions of the dilatation operator as

$$
\pi_{\phi}=\sqrt{\gamma}\left(\pi_{\left(\Delta_{-}\right)}+\cdots+\pi_{\left(\Delta_{+}\right)}+\cdots\right)
$$

where $\delta_{D} \pi_{(n)}=-n \pi_{(n)}$ for all $n .{ }^{4}$ This is simply a formal expansion at this point, as is (3.3), but the fact that $\phi$ and $\pi_{\phi}$ do admit the expansions (3.3) and (3.7) respectively, is a consequence of the equation of motion. Since $\pi_{\phi}=\sqrt{\gamma} \dot{\phi}$, one can insert the expansion (3.7) in (3.5) to obtain a formal expansion of the radial derivative in covariant functional operators of definite dilatation weight. Substituting this expansion for the radial derivative, together with the momentum expansion (3.7), into the equation of motion (3.2) and matching terms of equal dilatation weight one can determine iteratively all terms $\pi_{(n)}$ for $n<\Delta_{+}$as local functionals of $\phi$. The fact that these terms turn out to be local

\footnotetext{
${ }^{3}$ In general, one must sum over all induced fields on $\Sigma_{r}$, including the induced metric, $\gamma_{i j}$. In particular, the dilatation operator contains the term $\int_{\Sigma_{r}} d^{d} x 2 \gamma_{i j} \frac{\delta}{\delta \gamma_{i j}}$ and so it acts on the volume element on $\Sigma_{r}$ as $\delta_{D} \sqrt{\gamma}=d \sqrt{\gamma}$.

${ }^{4} \mathrm{~A}$ logarithmic term should be included in general to account for a possible conformal anomaly. In the presence of such an anomaly, $\pi_{\left(\Delta_{+}\right)}$transforms inhomogeneously under the dilatation operator [32].
} 
functionals of the induced field $\phi$ is the crucial ingredient which allows us to formulate the boundary value problem on the conformal boundary. In particular, we can write

$$
\int_{\Sigma_{r}} d^{d} x \sqrt{\gamma} \sum_{n<\Delta_{+}} \pi_{(n)} \delta \phi=-\delta S_{\mathrm{ct}}[\phi]
$$

where $S_{\mathrm{ct}}[\phi]$ is a local functional of $\phi$. From (3.4) then follows that if the local functional $S_{\text {ct }}$ on $\Sigma_{r}$ is added to the bulk action on $\mathcal{M}_{r}$, then a generic variation of the total action produces the boundary term ${ }^{5}$

$$
\delta\left(S+S_{\mathrm{ct}}\right)=\int_{\Sigma_{r}} d^{d} x \sqrt{\gamma} \pi_{\left(\Delta_{+}\right)} \delta \phi .
$$

Even though this might seem little different from the original expression (3.4), the difference is in fact fundamental: contrary to (3.4) the integrand in (3.9) is invariant under radial translations since $\delta_{D}\left(\sqrt{\gamma} \pi_{\left(\Delta_{+}\right)} \delta \phi\right)=\left(d-\Delta_{+}-\Delta_{-}\right) \sqrt{\gamma} \pi_{\left(\Delta_{+}\right)} \delta \phi=0$, where we have used $\delta_{D} \sqrt{\gamma}=d \sqrt{\gamma}$ (see footnote [3). If follows that we can now send $r \rightarrow \infty$ and any boundary condition formulated in terms of $\phi$ and the renormalized momentum $\pi_{\left(\Delta_{+}\right)}$will remain unchanged and meaningful in this limit.

Two comments are in order here. First, note that the counterterms we have defined via (3.8), and which were introduced only on the basis that they are required to make the variational problem on the conformal boundary well posed, are identical with the standard boundary counterterms that are traditionally added to make the on-shell action finite. Indeed, the fact that (3.9) has a finite limit as $r \rightarrow \infty$ implies that the renormalized on-shell action $S_{\text {ren }} \equiv\left(S+S_{\mathrm{ct}}\right)$, remains finite as the regulator is removed. The same local counterterms are therefore required to make the variational problem well posed and to remove the infra red divergences of the on-shell action. We would like to view the former, however, as the more fundamental property. Indeed, the divergences of the on-shell action are merely a manifestation of the fact that the variational problem is not formulated properly [31]. Of course, there is as usual a freedom of adding extra finite local terms to the counterterms $S_{\text {ct. }}$. In the case of Dirichlet boundary conditions this is the well known renormalization scheme dependence. As we will see below, however, the interpretation of this freedom in the dual field theory crucially depends on the boundary conditions. In particular, for boundary conditions other than Dirichlet, it does not correspond to a renormalization scheme dependence.

The second comment concerns some notation. It is very useful to introduce

$$
\widehat{\pi}_{\left(\Delta_{+}\right)} \equiv\left\{\begin{array}{l}
\lim _{r \rightarrow \infty} e^{\Delta_{+} r / l} \pi_{\left(\Delta_{+}\right)}, \Delta_{+}>d / 2, \\
\lim _{r \rightarrow \infty} r^{2} e^{d r / 2 l} \pi_{\left(\Delta_{+}\right)}, \Delta_{+}=d / 2,
\end{array}\right.
$$

which allows us to explicitly evaluate the limit $r \rightarrow \infty$ in (3.9) as

$$
\delta\left(S+S_{\mathrm{ct}}\right)=\int_{\partial \mathcal{M}} d^{d} x \sqrt{g_{(0)}} \widehat{\pi}_{\left(\Delta_{+}\right)} \delta \phi_{-} .
$$

\footnotetext{
${ }^{5}$ Terms of higher dilatation weight drop out in the limit $r \rightarrow \infty$.
} 


\begin{tabular}{|c|c|c|}
\hline & $J\left(\phi_{-}, \widehat{\pi}_{\left(\Delta_{+}\right)}\right)$ & $S_{J}\left[\phi_{-}, \widehat{\pi}_{\left(\Delta_{+}\right)}\right]$ \\
\hline Dirichlet & $J_{+}=\phi_{-}$ & $S_{+}=0$ \\
Neumann & $J_{-}=-\widehat{\pi}_{\left(\Delta_{+}\right)}$ & $S_{-}=-\int_{\partial \mathcal{M}} d^{d} x \sqrt{g_{(0)}} \phi_{-} \widehat{\pi}_{\left(\Delta_{+}\right)}$ \\
Mixed & $J_{f_{-}}=-\widehat{\pi}_{\left(\Delta_{+}\right)}-f^{\prime}\left(\phi_{-}\right)$ & $S_{f_{-}}=S_{-}+\int_{\partial \mathcal{M}} d^{d} x \sqrt{g_{(0)}}\left(f\left(\phi_{-}\right)-\phi_{-} f^{\prime}\left(\phi_{-}\right)\right)$ \\
\hline
\end{tabular}

Table 2: The three inequivalent boundary conditions for a scalar field in an AlAdS background, along with the corresponding boundary terms required to impose them. Notice that the Neumann boundary condition is a special case of the Mixed boundary condition, obtained by choosing the function $f\left(\phi_{-}\right)$to be identically zero.

The boundary value problem on the conformal boundary is then naturally formulated in terms of the two modes $\phi_{-}(x)$ and $\widehat{\pi}_{\left(\Delta_{+}\right)}$. Comparing the expansions (3.3) and (3.7), e.g. for $\Delta_{-} \neq \Delta_{+}$, one finds that $\widehat{\pi}_{\left(\Delta_{+}\right)}=-\left(\Delta_{+}-\Delta_{-}\right) \phi_{+}(x) / l+C\left[\phi_{-}(x)\right]$, where $C\left[\phi_{-}(x)\right]$ is a local functional of $\phi_{-}(x)$ depending on the space dimension as well as on the bulk dynamics. ${ }^{6}$ Interestingly, as we will later show, for the boundary conditions relevant to multi-trace deformations it turns out that $C\left[\phi_{-}(x)\right]$ vanishes identically - thus a posteriori justifying the use of $\phi_{+}(x)$ instead of the renormalized momentum in the literature. However, in general, it is $\widehat{\pi}_{\left(\Delta_{+}\right)}$and not the normalizable mode which has a well defined transformation under boundary Weyl transformations.

\subsection{Boundary conditions}

The expression (3.11) is our starting point for studying the possible boundary conditions on the conformal boundary. A boundary condition is in general a choice of a function, $J\left(\phi_{-}, \widehat{\pi}_{\left(\Delta_{+}\right)}\right)$, of the two independent modes, $\phi_{-}$and $\widehat{\pi}_{\left(\Delta_{+}\right)}$, that is kept fixed on the boundary. Note that we have now replaced $\phi_{+}$with $\widehat{\pi}_{\left(\Delta_{+}\right)}$, which as we discussed, is necessary in order for the boundary condition to be well defined on the conformal boundary. In order to impose the boundary condition $\delta J\left(\phi_{-}, \widehat{\pi}_{\left(\Delta_{+}\right)}\right)=0$, we need to add a suitable (finite) boundary term, $S_{J}\left[\phi_{-}, \widehat{\pi}_{\left(\Delta_{+}\right)}\right]$, to the action such that ${ }^{7}$

$$
\delta\left(S+S_{\mathrm{ct}}+S_{J}\right)=\int_{\partial \mathcal{M}} d^{d} x \sqrt{g_{(0)}} B_{J}\left(\phi_{-}, \widehat{\pi}_{\left(\Delta_{+}\right)}\right) \delta J\left(\phi_{-}, \widehat{\pi}_{\left(\Delta_{+}\right)}\right),
$$

where $B_{J}\left(\phi_{-}, \widehat{\pi}_{\left(\Delta_{+}\right)}\right)$is some function that depends on the choice of $J\left(\phi_{-}, \widehat{\pi}_{\left(\Delta_{+}\right)}\right)$.

A physical solution of the equations of motion, subject to the boundary condition defined by $J\left(\phi_{-}, \widehat{\pi}_{\left(\Delta_{+}\right)}\right)$, satisfies $J\left(\phi_{-}, \widehat{\pi}_{\left(\Delta_{+}\right)}\right)=0$. Note that this definition of 'physical

\footnotetext{
${ }^{6}$ We will see below that in the case of Dirichlet boundary conditions $\widehat{\pi}_{\left(\Delta_{+}\right)}$is identified via the AdS/CFT dictionary with the one-point function of the dual operator in the presence of an arbitrary source $\phi_{-}(x)$. The fact that the one-point function generically contains a non-linear but local functional, $C\left[\phi_{-}(x)\right]$, of the source was shown originally in 33$]$.

${ }^{7}$ The apparently alternative boundary condition $B_{J}\left(\phi_{-}, \widehat{\pi}_{\left(\Delta_{+}\right)}\right)=0$, is not acceptable in the context of the AdS/CFT correspondence. The reason is that such a boundary condition really reduces by half the degrees of freedom. In AdS/CFT, however, the boundary condition does halve the bulk degrees of freedom, but the lost half reappears as a source on the boundary.
} 
solutions' excludes solutions that describe single-trace deformations, which require a nonzero source. It follows that there are two qualitatively different universality classes of possible boundary conditions, depending on whether the mode $\phi_{-}(x)$ in the corresponding physical solutions is zero or not, which lead to different leading asymptotics for the physical solutions. $\phi_{-}(x)$ is zero in the physical solutions provided the source $J\left(\phi_{-}, \widehat{\pi}_{\left(\Delta_{+}\right)}\right)$is a function of $\phi_{-}$only. When $\phi_{-}(x)$ is non-zero in the physical solutions, then the relation $J\left(\phi_{-}, \widehat{\pi}_{\left(\Delta_{+}\right)}\right)=0$ determines $\widehat{\pi}_{\left(\Delta_{+}\right)}$as a function of $\phi_{-}(x)$. The three inequivalent choices of boundary conditions, along with the corresponding boundary term, $S_{J}\left[\phi_{-}, \widehat{\pi}_{\left(\Delta_{+}\right)}\right]$, that should be added to the action are listed in table 2.8

However, requiring that the (static) solutions subject to the boundary conditions in table 2 are perturbatively stable imposes restrictions on the allowed values of the scalar mass $m^{2}$. In particular, for Dirichlet boundary conditions, stability requires that the scalar mass satisfies the Breitenlohner-Freedman (BF) bound [22], $m^{2} l^{2} \geq-(d / 2)^{2}$, while if $\phi_{-}(x) \neq 0$ in the solution, i.e. for Neumann and Mixed boundary conditions, stability requires that the mass squared is in the range 222, 34, 35]

$$
-\left(\frac{d}{2}\right)^{2} \leq m^{2} l^{2} \leq-\left(\frac{d}{2}\right)^{2}+1
$$

We will later show that these stability conditions follow immediately from the requirement that there exists a stable holographic effective potential for the dual operator. Moreover, generically only one boundary condition will be consistent with supersymmetry once the scalar is embedded in some gauged supergravity [22, 36].

The mass constraint (3.13) for the Neumann or Mixed boundary conditions to be admissible has a remarkable and somewhat surprising consequence. Namely, it ensures that the local functional, $C\left[\phi_{-}(x)\right]$, which, as we pointed out above, distinguishes in general the renormalized momentum $\widehat{\pi}_{\left(\Delta_{+}\right)}$from the normalizable mode $\phi_{+}(x)$, vanishes identically. We will not give a general proof of this statement here, but one can understand it as follows. Generically, a non-zero $C\left[\phi_{-}(x)\right]$ can only arise if there are intermediate terms between the two modes, $\phi_{-}(x)$ and $\phi_{+}(x)$, in the asymptotic expansion (3.3). This can happen only if $\Delta_{+}-\Delta_{-}>\Delta_{-}$. However, (3.13) implies that for $d>2, \Delta_{+}-\Delta_{-} \leq \Delta_{-}$. Therefore, at least for $d>2$ and for a mass in the range (3.13), one has $\widehat{\pi}_{\left(\Delta_{+}\right)}=-\left(\Delta_{+}-\Delta_{-}\right) \phi_{+}(x) / l$ exactly, and hence, $\phi_{+}(x)$ is well defined on the boundary in this case.

\subsection{Solution of the boundary value problem}

The general solution of the boundary value problem with the boundary condition $\delta J\left(\phi_{-}, \widehat{\pi}_{\left(\Delta_{+}\right)}\right)=0$ consists in finding the most general regular solution of the bulk equations of motion as a functional of the arbitrary source $J\left(\phi_{-}, \widehat{\pi}_{\left(\Delta_{+}\right)}\right) \equiv J(x)$. This involves two steps:

\footnotetext{
${ }^{8}$ These boundary conditions exhaust all possible relations between $\widehat{\pi}_{\left(\Delta_{+}\right)}$and $\phi_{-}(x)$ in the physical solutions, and so all possible Hilbert spaces obtained by quantizing the scalar field. Nevertheless, there is an apparent redundancy in the choice of the source $J\left(\phi_{-}, \widehat{\pi}_{\left(\Delta_{+}\right)}\right)$. For example, $J=\phi_{-}$leads to the same Hilbert space as $J=\phi_{-}^{2}$. In the context of the AdS/CFT correspondence, this redundancy is mapped to an analogous redundancy in defining the generating functional of a given operator, and in particular in the choice of its source. Table 2 shows the standard minimal choices.
} 


\section{- Radial problem}

One solves the radial equation of motion exactly, imposing regularity in the interior. The result of this calculation is that (i) $\widehat{\pi}_{\left(\Delta_{+}\right)}$is determined as a non-local functional of $\phi_{-}{ }^{9}$ and (ii) the full bulk solution, $\phi\left(r, \phi_{-}(x)\right)$, is obtained as a non-local functional of $\phi_{-}(x)$.

\section{- Transverse problem}

To complete the solution of the boundary value problem, one needs to determine $\phi_{-}(x)$ as a functional of the arbitrary source $J(x)$. Having determined the functional $\widehat{\pi}_{\left(\Delta_{+}\right)}\left[\phi_{-}\right]$by solving the radial problem, this is achieved by solving the equation

$$
J\left(\phi_{-}, \widehat{\pi}_{\left(\Delta_{+}\right)}\left[\phi_{-}\right]\right)=J(x),
$$

for $\phi_{-}[J]$. For the boundary conditions in table 2, (3.14) reads

$$
\begin{aligned}
\phi_{-}=J(x), & \text { Dirichlet, } \\
-\widehat{\pi}_{\left(\Delta_{+}\right)}\left[\phi_{-}\right]=J(x), & \text { Neumann, } \\
-\widehat{\pi}_{\left(\Delta_{+}\right)}\left[\phi_{-}\right]-f^{\prime}\left(\phi_{-}\right)=J(x), & \text { Mixed. }
\end{aligned}
$$

Hence, the transverse problem is trivial for Dirichlet boundary conditions, but nontrivial for Neumann and Mixed boundary conditions. In all cases, inserting the resulting solution $\phi_{-}[J]$ back in the bulk solution of the radial problem we obtain the full solution $\phi\left(r, \phi_{-}[J(x)]\right)$.

Although the general prescription for solving the boundary value problem involves only these two simple steps, in practice there are very few cases where one is able to carry out either of these two steps. In particular, the bulk equations of motion are generically nonlinear, which makes the solution of the radial problem very difficult. On the other hand, if the function $f^{\prime}\left(\phi_{-}\right)$is non-linear, then the solution of the transverse problem (3.14) becomes very difficult too. In the next section, however, we will discuss a toy model for which it is possible to carry out the above prescription explicitly.

\subsection{The on-shell action and the AdS/CFT dictionary}

Assuming we have solved the boundary value problem with arbitrary sources to obtain the exact solution $\phi\left(r, \phi_{-}[J]\right)$, we can evaluate the on-shell action, $I[J]$. This involves three pieces: the bulk action, $S$, the covariant boundary counterterms, $S_{\mathrm{ct}}$, and the boundary term, $S_{J}$, defining the boundary condition. Namely,

$$
I[J]=\left.\left(S+S_{\mathrm{ct}}+S_{J}\right)\right|_{\phi},
$$

where the limit $r \rightarrow \infty$ is implicit. By construction, the value of the sum of these terms remains finite in this limit, and naturally, it is a functional of the source, $J$. The AdS/CFT

\footnotetext{
${ }^{9}$ This should in no way be confused with the boundary condition, which itself imposes another - algebraic - relation between the modes.
} 


\begin{tabular}{|c|c|c|c|}
\hline & Dirichlet & Neumann & Mixed \\
\hline$J$ & $J_{+} \equiv \phi_{-}$ & $J_{-} \equiv-\widehat{\pi}_{\left(\Delta_{+}\right)}$ & $J_{f_{-}} \equiv-\widehat{\pi}_{\left(\Delta_{+}\right)}-f^{\prime}\left(\phi_{-}\right)$ \\
$\sigma$ & $\widehat{\pi}_{\left(\Delta_{+}\right)}$ & $\phi_{-}$ & $\phi_{-}$ \\
$W[J]$ & $I_{+}\left[J_{+}\right]$ & $I_{-}\left[J_{-}\right]$ & $I_{f_{-}}\left[J_{f_{-}}\right]$ \\
$\Gamma[\sigma]$ & $I_{-}\left[-\widehat{\pi}_{\left(\Delta_{+}\right)}\right]$ & $I_{+}\left[\phi_{-}\right]$ & $I_{+}\left[\phi_{-}\right]+\int_{\partial \mathcal{M}} d^{d} x \sqrt{g_{(0)}} f\left(\phi_{-}\right)$ \\
$\left\langle T^{i j}\right\rangle=\frac{-2}{\sqrt{g_{(0)}} \frac{\delta W}{\delta g_{(0) i j}}}$ & $-2 \widehat{\pi}_{(d)}^{i j}$ & $-2 \widehat{\pi}_{(d)}^{i j}-\phi_{-} J_{-} g_{(0)} i j$ & $-2 \widehat{\pi}_{(d)}^{i j}-\left(f\left(\phi_{-}\right)+\phi_{-} J_{f_{-}}\right) g_{(0)}{ }^{i j}$ \\
$\left\langle T_{i}^{i}\right\rangle$ & $-\left(d-\Delta_{+}\right) J \sigma$ & $-\left(d-\Delta_{-}\right) J \sigma$ & $-\left(d-\Delta_{-}\right) J \sigma-d\left(f(\sigma)-\frac{\Delta_{-}}{d} \sigma f^{\prime}(\sigma)\right)$ \\
\hline
\end{tabular}

Table 3: The gravity/QFT dictionary.

dictionary, or more generally the gravity/quantum field theory dictionary, identifies the onshell action, $I[J]$, with the generating functional of connected correlation functions of the operator dual to the scalar field $\phi$. Namely,

$$
Z[J] \equiv e^{-W[J]}=\left\langle e^{-\int J \mathcal{O}}\right\rangle \approx e^{-I[J]}
$$

where the $\approx$ sign in the last equality means that the identification is understood in some certain limit, e.g. in the large $N$ limit, such that supergravity is a good approximation to the field theory dual. ${ }^{10}$ Since the on-shell action, $I[J]$, is identified with the generating functional of connected correlation functions, $W[J]$, its Legendre transform, $\Gamma[\sigma]$, given by

$$
\Gamma[\sigma]=W[J]-\int_{\partial \mathcal{M}} d^{d} x \sqrt{g_{(0)}} J \sigma,
$$

is the effective action of the dual operator, i.e. the generating functional of 1PI diagrams. In particular, the transverse problem (3.14) has a direct interpretation in the dual field theory as the 'gap equation' (2.4). From table 3 it is evident that, although the solution of the transverse problem is required in order to evaluate the generating functional, $W[J]$, for Neumann and Mixed boundary conditions (we have seen that the transverse problem is always trivial for Dirichlet boundary conditions), only the solution of the radial problem is necessary to evaluate the effective action, $\Gamma[\sigma]$.

In table 3 we summarize the identifications between the bulk and boundary quantities, according to the gravity/quantum field theory dictionary, for the three boundary conditions in table 2. Note that, since the on-shell action for Neumann boundary conditions is the Legendre transform of the on-shell action for Dirichlet boundary conditions (see the boundary term $S_{-}$in table 2), the effective action for Dirichlet boundary conditions is given

\footnotetext{
${ }^{10}$ We will not be specific about this limit since it depends crucially on the particular AdS/CFT duality. For example, in the most studied $\mathrm{AdS}_{5} / \mathrm{CFT}_{4}$ duality between $\mathcal{N}=4$ super Yang-Mills and Type II B string theory, the supergravity approximation involves not only the large $N$ limit, but also the large 't Hooft coupling limit. However, in the duality between M-theory on $\operatorname{AdS}_{4} \times S^{7}$ and the $\mathcal{N}=8$ SCFT in three dimensions, the supergravity approximation involves only the large $N$ limit as there is no other free parameter in this case.
} 
by the on-shell action for Neumann boundary conditions and vice versa. Moreover, the effective action for Mixed boundary conditions is given by the on-shell action for Dirichlet boundary conditions plus a term involving the function $f\left(\phi_{-}\right)$. Comparing the effective actions for Neumann and Mixed boundary conditions in table 3 with the expressions for the undeformed and deformed effective actions given in table 1, we reach the conclusion that the Mixed boundary conditions correspond to a multi-trace deformation of the QFT dual to the Neumann boundary conditions [1].

In the penultimate row of table 3 we show the renormalized VEV of the stress tensor of the dual theory in terms of the renormalized radial momentum, $\widehat{\pi}_{(d)}{ }^{i j}$, conjugate to the induced metric, $\gamma_{i j}$, (see appendix $\mathrm{A}$ ) for the three different boundary conditions. Note that the difference in these expressions for the VEV of the stress tensor is due to the boundary terms in table 2, which are required to impose each boundary condition. Using the fact that the bulk equations of motion determine that the trace of $\widehat{\pi}_{(d)}{ }^{i j}$ is related to the renormalized scalar momentum, $\widehat{\pi}_{\left(\Delta_{+}\right)}$, by $\widehat{\pi}_{(d)}=\Delta_{-} \widehat{\pi}_{\left(\Delta_{+}\right)} \phi_{-} / 2$ [32], these expressions allow us to write down the Conformal Ward identity for each boundary condition. These Ward identities are shown in the last row of table 3. We conclude that the Dirichlet and Neumann boundary conditions lead to a conformal field theory dual, since $\left\langle T_{i}^{i}\right\rangle=0$ for vanishing source, while the Mixed boundary condition leads to a conformal dual theory only if $f\left(\phi_{-}\right) \propto \phi_{-}^{d / \Delta_{-}}$. In that case, the Mixed boundary conditions describe a marginal multi-trace deformation of the CFT dual to the Neumann boundary conditions. Moreover, in the cases of a CFT dual, we see that the conformal dimension of the operator dual to the scalar field is $\Delta_{+}$for Dirichlet boundary conditions and $\Delta_{-}$for Neumann and Mixed boundary conditions. This is as expected, since the leading asymptotic behavior of the physical solutions is determined by $\Delta_{+}$for Dirichlet boundary conditions (i.e. $\phi_{-}=0$ ), but by $\Delta_{-}$for Neumann and Mixed boundary conditions $\left(\phi_{-} \neq 0\right)$.

\section{Toy model}

The boundary value problem can be solved in complete generality, following the prescription outlined above, for a free scalar field in a fixed AdS background with the action

$$
S=\int d^{d+1} x \sqrt{g}\left(\frac{1}{2} g^{\mu \nu} \partial_{\mu} \phi \partial_{\nu} \phi+\frac{1}{2} m^{2} \phi^{2}\right) .
$$

The metric here is (3.1) with $\gamma_{i j}=e^{2 r / l} \delta_{i j}$, which is the metric of exact $\operatorname{AdS}_{d+1}$ (more precisely $\mathbb{H}_{d+1}$ ) in the upper half plane coordinates. The equation of motion is of course the Klein-Gordon equation (3.2). Even though the fact that the bulk equation of motion is linear means that it is possible to solve the radial problem exactly, the transverse problem remains in general intractable, except for certain linear boundary conditions.

\subsection{General solution with linear boundary conditions}

Counterterms. In order to compute the renormalized momentum, as well as the on-shell action, we need to know the covariant boundary counterterms. This is done, as we discussed 
above, by inserting the covariant expansion (3.7) of the canonical momentum, and of the radial derivative (3.5), into the equation of motion (3.2). This iteratively determines 37, 38]

$$
\begin{aligned}
\pi_{\left(\Delta_{-}\right)} & =-\frac{\Delta_{-}}{l} \phi, \\
\pi_{\left(\Delta_{-}+2\right)} & =\frac{-l \square_{\gamma} \phi}{\left(d-2 \Delta_{-}-2\right)}, \\
\pi_{\left(\Delta_{-}+4\right)} & =-\frac{l^{3}\left(-\square_{\gamma}\right)^{2} \phi}{\left(d-2 \Delta_{-}-2\right)^{2}\left(d-2 \Delta_{-}-4\right)}, \quad \ldots
\end{aligned}
$$

This procedure breaks down at order $\Delta_{+}$leaving $\pi_{\left(\Delta_{+}\right)}$undetermined. From (3.8) now we see that the counterterms are obtained by integrating these momenta with respect to the scalar field. This gives [30, 37, 38]

$$
S_{\mathrm{ct}}=\frac{1}{2} \int_{\Sigma_{r}} d^{d} x \sqrt{\gamma} \phi\left(-\frac{\Delta_{-}}{l} \phi+\frac{-l \square_{\gamma} \phi}{\left(d-2 \Delta_{-}-2\right)}-\frac{l^{3}\left(-\square_{\gamma}\right)^{2} \phi}{\left(d-2 \Delta_{-}-2\right)^{2}\left(d-2 \Delta_{-}-4\right)}+\cdots\right) .
$$

If we restrict to the mass range (3.13), which is necessary in order to consider Neumann and Mixed boundary conditions, then only the first term in (4.3) is relevant since $\Delta_{+} \leq \Delta_{-}+2$ in this case. For Dirichlet boundary conditions, however, more terms must be kept in general.

Radial problem. The most general solution of the radial part of the equation of motion (3.2) that is regular in the interior can be written in two equivalent forms. The first is an exact expression for the canonical momentum as a covariant functional of the induced field $\phi$, namely [37, 38]

$$
\pi_{\phi}[\phi]=\sqrt{\gamma} \dot{\phi}=\sqrt{\gamma} \frac{1}{l}\left(-\frac{d}{2}-l \sqrt{-\square_{\gamma}} \frac{K_{\nu}^{\prime}\left(l \sqrt{-\square_{\gamma}}\right)}{K_{\nu}\left(l \sqrt{-\square_{\gamma}}\right)}\right) \phi
$$

where $\nu=\left(\Delta_{+}-\Delta_{-}\right) / 2$ and $K_{\nu}$ is the modified Bessel function that is regular for large argument. Fourier transforming (4.4) and solving the resulting linear first order equation gives the second form

$$
\phi\left(r, \phi_{-}(x)\right)=\frac{l^{\nu}}{2^{\nu-1} \Gamma(\nu)} e^{-d r / 2 l} \int \frac{d^{d} p}{(2 \pi)^{d}} \tilde{\phi}_{-}(p) p^{\nu} K_{\nu}\left(l p e^{-r / l}\right) e^{i p \cdot x},
$$

where $\tilde{\phi}_{-}(p)$, which appears as the integration constant of the first order equation, is the Fourier transform of an arbitrary function $\phi_{-}(x)$. Using the asymptotic form of the Bessel function, $K_{\nu}\left(l p e^{-r / l}\right) \sim 2^{\nu-1} l^{-\nu} \Gamma(\nu) e^{\nu r / l} p^{-\nu}$, as $r \rightarrow \infty$, we see that $\phi \sim e^{-\Delta_{-} r / l} \phi_{-}(x)$ asymptotically, in agreement with (3.3).

The form (4.4) is particularly useful because, by expanding the Bessel function for small argument, one automatically obtains the covariant expansion (3.7), but now including the renormalized momentum $\pi_{\left(\Delta_{+}\right)}$. For $0<\nu<1$, which corresponds to the mass 
range $^{11}(3.13)$, this is

$$
\widehat{\pi}_{\left(\Delta_{+}\right)}\left[\phi_{-}\right]=\left(\frac{l}{2}\right)^{2 \nu-1} \frac{\Gamma(1-\nu)}{\Gamma(\nu)}(-\square)^{\nu} \phi_{-} .
$$

This non-local relation is the essential ingredient in order to address the transverse problem.

Transverse problem. Since the transverse problem (3.14) is trivial for Dirichlet boundary conditions, the solution of the radial problem, (4.4) or (4.5), is sufficient to evaluate the on-shell action. The result is shown in the second column of table $\mathrm{A}$. From table 3 follows that this also allows us to evaluate the effective action for Neumann and Mixed boundary conditions, since it is directly related to the on-shell action for Dirichlet boundary conditions. For the Mixed boundary conditions then, which include the Neumann as a special case, the effective action is

$$
\Gamma_{f_{-}}\left[\phi_{-}\right]=\int d^{d} x\left(\frac{1}{2}\left(\frac{l}{2}\right)^{2 \nu-1} \frac{\Gamma(1-\nu)}{\Gamma(\nu)} \phi_{-}(-\square)^{\nu} \phi_{-}+f\left(\phi_{-}\right)\right) .
$$

Functionally differentiating this with respect to the VEV, we explicitly see that the transverse problem (3.14), which, using (4.6), takes the form

$$
-\left(\frac{l}{2}\right)^{2 \nu-1} \frac{\Gamma(1-\nu)}{\Gamma(\nu)}(-\square)^{\nu} \phi_{-}-f^{\prime}\left(\phi_{-}\right)=J(x),
$$

is nothing but the gap equation (2.4).

Solving (4.8) for $\phi_{-}[J]$ is in general not possible unless $f\left(\phi_{-}\right)=\xi \phi_{-}^{2}$, for some constant $\xi$. As we have seen, this corresponds to a double-trace deformation of the dual theory. With this special choice of Mixed boundary conditions, the transverse problem is easily solved and the on-shell action can be evaluated. The results are shown in table 6 . Moreover, inserting the expressions for $\phi_{-}[J]$ given in table 1 in the solution (4.5), we obtain the full solution to the boundary value problem with the corresponding linear boundary conditions. Note that the expression for the on-shell action in the presence of a double-trace deformation shown in table 1 is in complete agreement with the corresponding expressions in e.g. [9], but ours are manifestly cut-off independent.

\subsection{Vacua with non-linear boundary conditions}

Even though one cannot solve equation (4.8) in general for non-linear boundary conditions, we can still find exact solutions of the corresponding sourceless equation. The classification of such 'vacuum'12 solutions is essential before one can solve (4.8) perturbatively in the source, $J(x)$, around each vacuum. We will not attempt a classification of the vacuum

\footnotetext{
${ }^{11}$ The endpoints, $\nu=0,1$, correspond respectively to the cases where the BF bound and the unitarity bound are saturated. Whenever $\nu$ is an integer the Bessel function involves logarithms. These cases can be treated similarly. See e.g. 30, 37, 38] for the case of Dirichlet boundary conditions, where logarithms also appear.

${ }^{12}$ By the term 'vacuum' we do not necessarily refer to a constant or time independent solution $\phi_{-}$. Any solution to the sourceless equation (3.14) will be called a vacuum.
} 


\begin{tabular}{|c|c|c|c|}
\hline & Dirichlet & Neumann & Mixed \\
\hline$J$ & $J_{+} \equiv \phi_{-}$ & $J_{-} \equiv-\widehat{\pi}_{\left(\Delta_{+}\right)}$ & $J_{f_{-}} \equiv-\widehat{\pi}_{\left(\Delta_{+}\right)}-2 \xi \phi_{-}$ \\
$\phi_{-}[J]$ & $J_{+}$ & $-2^{2 \nu-1} \frac{l \Gamma(\nu)}{\Gamma(1-\nu)}\left(-l^{2} \square\right)^{-\nu} J_{-}$ & $-\left(2 \xi+\left(\frac{1}{2}\right)^{2 \nu-1} \frac{\Gamma(1-\nu)}{l \Gamma(\nu)}\left(-l^{2} \square\right)^{\nu}\right)^{-1} J_{f_{-}}$ \\
$I[J]$ & $\frac{\Gamma(1-\nu)}{l 2^{2 \nu} \Gamma(\nu)} \int J_{+}\left(-l^{2} \square\right)^{\nu} J_{+}$ & $-\frac{l 2^{2 \nu} \Gamma(\nu)}{4 \Gamma(1-\nu)} \int J_{-}\left(-l^{2} \square\right)^{-\nu} J_{-}$ & $-\frac{1}{4} \int J_{f_{-}}\left(\xi+\frac{\Gamma(1-\nu)}{l 2^{2 \nu} \Gamma(\nu)}\left(-l^{2} \square\right)^{\nu}\right)^{-1} J_{f_{-}}$ \\
\hline
\end{tabular}

Table 4: The solutions of the transverse problem and the on-shell action for all three linear boundary conditions. $\xi$ is an arbitrary constant corresponding to the deformation parameter of the double-trace deformation.

solutions for various choices of $f\left(\phi_{-}\right)$here. Instead, we now give two examples of nontrivial vacua which are closely related to the vacua we will construct later on for more realistic interacting theories.

Constant VEV. For any choice of the function $f\left(\phi_{-}\right)$, a constant, $\phi_{-}^{*}$, that extremizes $f\left(\phi_{-}\right)$, i.e. $f^{\prime}\left(\phi_{-}^{*}\right)=0$, is a solution of the sourceless equation (4.8). Indeed, from (4.7) we know that $f_{-}(\phi)$ is the effective potential of the dual theory. The Fourier transform of a constant $\phi_{-}^{*}$ is a delta function in momentum space, $\tilde{\phi}_{-}^{*}=\phi_{-}^{*}(2 \pi)^{d} \delta^{(d)}(p)$. Inserting this into the general solution (4.5) we obtain an exact solution of the bulk equation of motion satisfying Mixed boundary conditions. Namely,

$$
\phi\left(r, \phi_{-}^{*}\right)=\frac{l^{\nu}}{2^{\nu-1} \Gamma(\nu)} e^{-d r / 2 l} \phi_{-}^{*} \lim _{p \rightarrow 0} p^{\nu} K_{\nu}\left(l p e^{-r / l}\right) e^{i p \cdot x}=e^{-\Delta_{-} r / l} \phi_{-}^{*} .
$$

Instantons. Non-constant solutions can also be found, at least for certain choices of the potential $f\left(\phi_{-}\right)$. To look for non-constant solutions, however, we need an explicit representation of the operator $(-\square)^{\nu}$. It is in fact easier to find a representation of the inverse of this operator, which has the integral representation

$$
(-\square)^{-\nu} \Phi(x)=\frac{\Gamma\left(\frac{d}{2}-\nu\right)}{2^{2 \nu} \pi^{d / 2} \Gamma(\nu)} \int d^{d} y \frac{\Phi(y)}{|x-y|^{d-2 \nu}}, \quad \nu>0 .
$$

The sourceless equation (4.8) can then be written as the integral equation

$$
\phi_{-}(x)+\frac{\Gamma\left(\frac{d}{2}-\nu\right)}{2 \pi^{d / 2} l^{2 \nu-1} \Gamma(1-\nu)} \int d^{d} y \frac{f^{\prime}\left(\phi_{-}(y)\right)}{|x-y|^{d-2 \nu}}=0 .
$$

We now look for solutions of the form

$$
\phi_{-}(x)=\frac{b}{|x|^{c}},
$$

for a potential of the form $f\left(\phi_{-}\right)=\xi \phi_{-}^{\omega}$, where $b, c, \xi, \omega$ are constants. Inserting these into (4.11) and Fourier transforming determines that such a solution exists only if $\omega>2$, 
i.e. provided the boundary condition is non-linear, and also only if $\xi<0$, i.e. when the effective potential is unbounded from below. Moreover, $c$ and $b$ are given by

$$
c=\frac{2 \nu}{\omega-2}, \quad b^{\omega-2}=\frac{2 l^{2 \nu-1} \Gamma(1-\nu) \Gamma\left(\frac{d}{2}-\frac{\nu}{\omega-2}\right) \Gamma\left(\frac{(\omega-1) \nu}{\omega-2}\right)}{\omega|\xi| \Gamma(\nu) \Gamma\left(\frac{\nu}{\omega-2}\right) \Gamma\left(\frac{d}{2}-\frac{(\omega-1) \nu}{\omega-2}\right)} .
$$

Inserting (4.12) into (4.5) we obtain the exact bulk solution

$$
\begin{aligned}
& \phi\left(r, \phi_{-}(x)\right)= \\
& b l^{-\frac{2 \nu}{\omega-2}} \frac{\Gamma\left(\frac{d}{2}-\frac{\nu}{\omega-2}\right) \Gamma\left(\frac{(\omega-1) \nu}{\omega-2}\right)}{\Gamma\left(\frac{d}{2}\right) \Gamma(\nu)} e^{-\left(\frac{d}{2}-\frac{\omega \nu}{\omega-2}\right) \frac{r}{l}} F\left(\frac{\nu}{\omega-2}, \frac{(\omega-1) \nu}{\omega-2} ; \frac{d}{2} ;-\frac{1}{l^{2}} e^{2 r / l} x^{2}\right) .
\end{aligned}
$$

The asymptotic form of this solution is

$$
\phi\left(r, \phi_{-}(x)\right) \sim\left(\frac{b}{|x|^{c}}\right) e^{-\Delta_{-} r / l}+\frac{l}{2 \nu} \omega \xi\left(\frac{b}{|x|^{c}}\right)^{\omega-1} e^{-\Delta_{+} r / l},
$$

i.e. $\phi_{+}=\frac{l}{2 \nu} \omega \xi \phi_{-}^{\omega-1}$. Since $\widehat{\pi}_{\left(\Delta_{+}\right)}=-2 \nu \phi_{+}(x) / l$, it follows that (4.14) indeed satisfies Mixed boundary conditions with $f\left(\phi_{-}\right)=\xi \phi_{-}^{\omega}$, for $J_{f_{-}}=-\widehat{\pi}_{\left(\Delta_{+}\right)}-f^{\prime}\left(\phi_{-}\right)=0$.

This Euclidean solution is in fact analogous to the instanton solution found in 24] for a scalar field conformally coupled to four-dimensional gravity, which we will revisit and generalize below. As for the instanton solution of [24, (4.14) exists only when the effective potential is unbounded from below, i.e $\xi<0$, which means that the deformation induces an instability in the boundary CFT. In particular, (4.14) describes the decay process of the trivial vacuum at $\phi_{-}=0$ to an instability region at $\phi_{-} \rightarrow \infty$. The decay rate is given by [39]

$$
\mathcal{P} \propto e^{-\left.\Gamma_{f}\right|_{\text {inst. }},}
$$

where the value of the effective action (4.7) evaluated on the instanton solution is

$$
\left.\Gamma_{f}\right|_{\text {inst. }}=\frac{d(\omega-2)|\xi|}{2\left(d-\frac{2 \nu \omega}{\omega-2}\right)} b^{\omega} \operatorname{Vol}\left(S^{d}\right)
$$

\section{Effective action from Hamilton's characteristic function}

The free scalar field in a fixed AdS background is a useful example as a boundary value problem in AlAdS spaces, but, in the context of the AdS/CFT correspondence, it can only give information on the dual $\mathrm{CFT}$ at the conformal vacuum. As soon as the scalar field acquires a non-zero VEV conformal invariance is broken and one must couple the scalar to dynamical gravity in order to study holographically the dual field theory. In particular, although the conformal vacuum generically remains a vacuum of the dual theory when the latter is deformed by a multi-trace deformation, the deformation may not only destabilize the conformal vacuum, but also it will generically introduce new vacua. AdS/CFT relates the problem of stability of the conformal vacuum under multi-trace deformations to the stability of AdS under the corresponding boundary conditions on the dual bulk fields. 
Both the non-perturbative stability of the conformal vacuum and the possible appearance of new vacua due to a generic multi-trace deformation can be addressed if one knows the effective action for the deforming operator in the dual field theory. In the large $N$ limit, the AdS/CFT dictionary relates the effective action of the boundary theory to the on-shell supergravity action. More specifically, the on-shell action with Dirichlet boundary conditions is related to the effective action of the theory with Neumann or Mixed boundary conditions and vice versa (see table 3). However, computing the on-shell supergravity action non-perturbatively in the scalar field - which is required if non-perturbative stability is to be addressed - is of course not an easy task. Even though the boundary metric $g_{(0) i j}$ can be set to a fixed value for this computation, since we are only interested in the back-reaction of the scalar field on the bulk metric, the bulk equations remain highly non-linear and generically too difficult to solve. Nevertheless, there is a systematic way to approximate the effective action away from the conformal vacuum. Since, for conformal boundary conditions, conformal invariance is only broken spontaneously by the non-zero VEV of the scalar field, in a vacuum of non-zero scalar VEV the two-point function of the scalar operator always contains a massless pole, corresponding to the Goldstone boson of spontaneously broken scale invariance, which dominates the two-point function for small momenta. This massless pole gives, via the Legendre transform, a standard quadratic kinetic term in the effective action for the VEV of the scalar operator. It follows that, at least for conformal boundary conditions, the effective action admits a derivative expansion away from the conformal vacuum. But since we know that a generic multi-trace deformation simply modifies the effective potential in the large $N$ limit, the above argument implies that the effective action always admits a derivative expansion away from the conformal vacuum. As we will now see, this fact allows us to systematically construct the effective action to any order in derivatives, although the computation quickly becomes tedious.

According to standard practice, to evaluate the renormalized on-shell action one needs to compute two related - yet distinct - quantities. Namely, the covariant boundary counterterms, $S_{\text {ct }}$, and the regularized action, $I_{r}$ (see (A.6)). Let us take the opportunity here to emphasize that the split of the computation into two separate computations of $S_{\text {ct }}$ and $I_{r}$ is only an artificial split reflecting the difference in technical difficulty in computing these two quantities. While the counterterms can always be computed in full generality by some version of holographic renormalization, the computation of $I_{r}$ is far more difficult and usually requires some approximation. However, if one were able to compute $I_{r}$ exactly, then the counterterms, and hence the renormalized on-shell action, can be immediately deduced by expanding $I_{r}$ in eigenfunctions of the dilatation operator. In fact, we saw such an example in section 1 , where the exact expression for the momentum (4.4) enabled us to simply read off the renormalized momentum (4.6). But the advantage of the expansion in eigenfunctions of the dilatation operator is that it works equally well even when $I_{r}$ is only known in some approximation, be that a small source expansion or a derivative expansion. In particular, instead of the traditional 3-step approach: 1 . compute $S_{\mathrm{ct}}$ in full generality, 2. compute $I_{r}$ in some approximation 3. reduce the counterterms in the approximation used for evaluating $I_{r}$, we will use the more efficient 2-step approach: 1 . compute $I_{r}$ in whatever approximation is suitable 2. extract the renormalized part of $I_{r}$ by 
expanding it in eigenfunctions of the dilatation operator and keeping terms of weight zero. This technique was applied to compute renormalized correlation functions in 40], which involved evaluating $I_{r}$ in a small source approximation. It can equally well be applied in the case of a derivative expansion of $I_{r}$, which is the relevant approximation here. Hence, we simply need to worry about the evaluation of the regularized on-shell action $I_{r}$, as the renormalized action can effectively be read off $I_{r}$.

The most direct way to compute the regularized on-shell action is via the radial Hamilton-Jacobi formalism [29. ${ }^{13}$ This amounts to solving the two functional equations resulting from inserting the radial momenta, given as functional derivatives of the regularized on-shell action with respect to the induced fields on $\Sigma_{r}$ as in (A.7), in the Hamiltonian and momentum constraints (A.8). Although the resulting functional differential equations are generically too complicated to solve, their virtue is that they directly determine the regularized on-shell action. In practice, this approach is useful if one is able to write down the most general ansatz for $I_{r}$ in a certain approximation, or if one is interested in the 'minisuperspace' of a certain class of solutions, since then the Hamilton-Jacobi equation can be simplified drastically. Although the local part of $I_{r}$, which will be removed by the boundary counterterms, always takes the form of a derivative expansion and so can be determined by an obvious ansatz as in [29], here we are interested in the non-local part of the regularized action. Finding a suitable ansatz for this non-local part is a much more difficult question. However, this crucially depends on the physical interpretation of the regularized action. In particular, while a local derivative expansion of the non-local part of $I_{r}$ is useless if $I_{r}$ is identified with the generating functional of the dual operator, since a derivative expansion only gives contact terms in the corresponding correlation functions, it does makes sense to expand the non-local part of $I_{r}$ in a derivative expansion if it is interpreted as the effective action of the dual operator. Indeed, we have argued above that the effective action admits such a local approximation away from the conformal vacuum.

Our method for evaluating the regularized on-shell action then consists of two complementary computations, involving different but not mutually exclusive approximations. First, we start from the undeformed CFT on a (nearly) flat boundary, in which case the regularized action in the two-derivative approximation takes the form ${ }^{14}$

$$
I_{r}=\int_{\Sigma_{r}} d^{d} x \sqrt{\gamma}\left(W(\phi)+Z(\phi) R[\gamma]+\frac{1}{2} M(\phi) \gamma^{i j} \partial_{i} \phi \partial_{j} \phi\right)
$$

where $W(\phi), Z(\phi)$ and $M(\phi)$ are functions of the scalar field to be determined. Differentiating (5.1) with respect to the induced metric and the scalar field yields respectively the

\footnotetext{
${ }^{13}$ It is worth pointing out that the interpretation of the Hamilton-Jacobi equation for the on-shell action as the Callan-Symanzik equation for the generating functional of the dual operator in 29] has an obvious analogue when the AdS/CFT dictionary identifies the on-shell action with the effective action of the dual operator.

${ }^{14}$ See 41] for a systematic approach to solving the Hamilton-Jacobi equation in a derivative (long wavelength) expansion in a similar context.
} 
momenta (see A.7)

$$
\begin{aligned}
& \pi^{i j}=\sqrt{\gamma}\{(\left.Z^{\prime \prime}-\frac{1}{2} M\right) \partial^{i} \phi \partial^{j} \phi+Z^{\prime} D^{i} D^{j} \phi-Z R^{i j} \\
&\left.+\frac{1}{2} \gamma^{i j}\left[W+Z R-2 Z^{\prime} \square_{\gamma} \phi+\left(\frac{1}{2} M-2 Z^{\prime \prime}\right) \partial^{k} \phi \partial_{k} \phi\right]\right\}, \\
& \pi_{\phi}=\sqrt{\gamma}\left\{W^{\prime}+Z^{\prime} R-\frac{1}{2} M^{\prime} \partial^{k} \phi \partial_{k} \phi-M \square_{\gamma} \phi\right\} .
\end{aligned}
$$

These momenta automatically satisfy the momentum constraint in (A.8), which is simply a consequence of the invariance of (5.1) under $\Sigma_{r}$ diffeomorphisms. Inserting the momenta (5.2) into the Hamiltonian constraint in (A.8) leads to a set of ordinary differential equations for the functions $W(\phi), Z(\phi)$ and $M(\phi)$, which of course depend on the form of the Hamiltonian. Having determined these functions, the regularized action is expanded in eigenfunctions of the dilatation operator (which in this case amounts to a simple Taylor expansion of $W(\phi), Z(\phi)$ and $M(\phi))$ and the term of zero dilatation weight, corresponding to the renormalized action, is isolated. The structure of the resulting renormalized twoderivative effective action is largely universal as it is determined by conformal invariance. However, there is a free parameter which is left undetermined both by the above procedure and by conformal invariance. This raises the question as to what is the significance of this parameter and how it can be fixed. Another question that is left unanswered by the above computation is how one can evaluate the effective action with general boundary conditions, i.e. the effective action of the deformed CFT. The diffeomorphism invariance of the ansatz (5.1) implicitly assumes that conformally invariant boundary conditions are imposed, which is reflected in the fact that the renormalized action one obtains from this ansatz is conformally invariant. However, a generic boundary condition corresponding to a relevant deformation will break this invariance. So, how then are relevant deformations accommodated in the Hamilton-Jacobi formalism? The answer to both questions is clearly that the ansatz (5.1) is too restrictive.

Instead of looking for a suitable generalization of the ansatz (5.1), however, we will try to solve the Hamilton-Jacobi equation exactly - without any ansatz - but in a zeroderivative approximation. That is, assuming the metric and the scalar are functions of the radial coordinate only. Clearly, this determines the most general effective potential and hence it must account for any multi-trace deformation. Moreover, this calculation can in principle be done for any boundary, not just a (nearly) flat boundary as was assumed in (5.1). The result will be the exact effective potential on the given boundary. As we will see, this answers the first question, since if one is able to evaluate the effective potential on, say, the sphere, then expanding this for small curvature and comparing with the result of the previous calculation based on the ansatz (5.1) fixes the undetermined parameter in the two derivative effective action.

Interestingly, upon a choice of a boundary manifold, the zero-derivative approximation amounts to looking at the 'minisuperspace' of certain domain-wall like solutions. In particular, for a boundary that cannot be written as the direct product of two sub-manifolds, 
this approximation corresponds to looking for domain walls of the form

$$
d s^{2}=d r^{2}+e^{2 A(r)} g_{(0) i j}(x) d x^{i} d x^{j}, \quad \phi=\phi(r),
$$

where $g_{(0) i j}(x)$ is a metric independent of the radial coordinate $r$. The equations of motion require that $g_{(0) i j}(x)$ is maximally symmetric, $R\left[g_{(0)}\right]_{i j}=\frac{1}{d} R\left[g_{(0)}\right] g_{(0) i j}$, and has locally constant scalar curvature, $R\left[g_{(0)}\right]=k d(d-1) / l^{2}$, where $k=0, \pm 1$. The hypersurface $\Sigma_{r}$ then can be $S^{d}, \mathbb{R}^{d}$ or $\mathbb{H}_{d}$, or a quotient of these by a discrete subgroup of their isometry group. Replacing the induced metric $\gamma_{i j}$ as a dynamical field by the warp factor, $A(r)$, and the canonical momentum $\pi^{i j}$ by the momentum, $\pi_{A}$, conjugate to $A$, which is defined via $\pi^{i j} \delta \gamma_{i j}=\pi_{A} \delta A$, reduces the Hamilton-Jacobi equation to a PDE for the effective potential as a function of the two variables $A$ and $\phi$. This can then be viewed as the Hamilton-Jacobi equation for a standard classical mechanics problem for the generalized coordinates $A$ and $\phi$. As is well known from Hamilton-Jacobi theory [42], the general solution of the equations of motion, i.e. the most general solution of the form (5.3) in this case, can be obtained from any complete integral of the Hamilton-Jacobi equation, which in this case contains one arbitrary integration constant. ${ }^{15}$ However, the Hamilton-Jacobi equation admits more than one complete integrals. In fact, the general solution of the Hamilton-Jacobi equation contains an arbitrary function - not just a constant. As we will show below, this freedom in choosing a complete integral for the Hamilton-Jacobi equation in the zero-derivative approximation corresponds precisely to the choice of boundary conditions.

Put together then, the two-derivative effective action with conformal boundary conditions based on the ansatz (5.1), and the 'minisuperspace' approximation of the HamiltonJacobi equation to solutions of the form (5.3), completely determine the two-derivative effective action of the dual operator on $S^{d}, \mathbb{R}^{d}$ or $\mathbb{H}_{d}$ (or any of their quotients) and for any boundary conditions. Moreover, this computation can be generalized to other boundaries too. For example, one can compute the two-derivative effective action on $\mathbb{R} \times S^{d-1}$ by solving the Hamilton-Jacobi equation for metrics of the form

$$
d s^{2}=d r^{2}+e^{2 A(r)} d \tau^{2}+e^{2 B(r)} d \Omega_{d-1}^{2},
$$

instead of the domain walls 5.3 ).

\section{Minimal coupling}

In this section we apply the above method to the system of a single scalar field minimally coupled to gravity, which is described by the action

$$
S=\int_{\mathcal{M}} d^{d+1} \sqrt{g}\left(-\frac{1}{2 \kappa^{2}} R+\frac{1}{2} g^{\mu \nu} \partial_{\mu} \phi \partial_{\nu} \phi+V(\phi)\right),
$$

where $\kappa^{2}=8 \pi G_{d+1}$ is the gravitational constant. ${ }^{16}$ An action of this form generically arises as a consistent truncation of some gauged supergravity, ${ }^{17}$ but we need not be specific about

\footnotetext{
${ }^{15}$ This is because there is no explicit dependence on the radial 'time'. The characteristic function for $n$ variables contains $n-1$ arbitrary constants 42 .

${ }^{16}$ See appendix A for a detailed discussion of the variational problem and a derivation of the appropriate boundary terms.

${ }^{17}$ Of course, the 'consistency' of the truncation must be checked at the level of the equations of motion.
} 
the embedding of (6.1) into a particular gauged supergravity at this point. We will later give an example of a potential which allows this action to be embedded into $\mathcal{N}=8$ gauged supergravity in four dimensions and, hence, be uplifted to 11-dimensional supergravity, but we would like to keep the discussion as general as possible here.

The action (6.1) possesses an AdS vacuum of radius $l$ provided the potential has a negative extremum at $\phi=\phi_{o}$, such that $V^{\prime}\left(\phi_{o}\right)=0, V\left(\phi_{o}\right)=-d(d-1) / 2 \kappa^{2} l^{2}$. It follows that, in the vicinity of this extremum, the potential takes the form

$$
V(\phi)=-\frac{d(d-1)}{2 \kappa^{2} l^{2}}+\frac{1}{2} m^{2}\left(\phi-\phi_{o}\right)^{2}+o\left(\left(\phi-\phi_{o}\right)^{2}\right),
$$

where $m$ is the mass of the scalar field. Note that, unless the potential is exactly constant, the location of the extremum is at some fixed value, $\phi_{o}$, which can be set to zero by a redefinition of the scalar field. Moreover, if the mass vanishes, then the potential must be constant, or else the equations of motion eliminate the mode $\phi_{-}(x)$ in the expansion (3.3). Below we will focus on masses in the range $-(d / 2)^{2} \leq m^{2} l^{2}<0$, excluding the case of a constant potential.

\subsection{Two-derivative effective action for conformal boundary conditions}

Our first task is to determine the renormalized on-shell action corresponding to conformal boundary conditions using the ansatz (5.1). Using the Hamiltonian (A.9), which is relevant for the action (6.1), the Hamiltonian constraint leads to three independent equations for the functions $W(\phi), Z(\phi)$ and $M(\phi)$. Namely,

$$
\begin{gathered}
V(\phi)=\frac{1}{2}\left(W^{\prime 2}-\frac{d \kappa^{2}}{d-1} W^{2}\right), \\
W^{\prime} Z^{\prime}-\kappa^{2} \frac{d-2}{d-1} W Z+\frac{1}{2 \kappa^{2}}=0, \\
M=2 \kappa^{2} \frac{W}{W^{\prime}} Z^{\prime} .
\end{gathered}
$$

The last equation gives explicitly the function $M(\phi)$ in terms of $W$ and $Z$. Moreover, the second equation is a linear equation for $Z$, whose solution in terms of $W$ is

$$
Z(\phi)=-\frac{1}{2 \kappa^{2}} Z_{o} \int^{\phi} \frac{d \bar{\phi}}{W^{\prime} Z_{o}}, \quad Z_{o}(\phi) \equiv \exp \left(\kappa^{2} \frac{d-2}{d-1} \int^{\phi} d \bar{\phi} \frac{W}{W^{\prime}}\right) .
$$

The regularized two-derivative effective action is therefore determined by the non-linear equation (6.3) for the function $W(\phi)$, which we will call 'fake superpotential' for reasons that will become clear later on. Equation (6.3) can be transformed [32] into the standard form of Abel's equation of the first kind 43]. Although its general solution is not known for an arbitrary scalar potential $V(\phi)$, for certain choices of the potential it falls into some of the known integrability classes of Abel's equation and it can be solved exactly. We will discuss such an example below, but in order to determine the renormalized action we need not solve (6.3) exactly. Indeed, we will now show that some general features of the solutions of equation (6.3) are sufficient for this purpose. 


\begin{tabular}{|c|l|}
\hline$m^{2} l^{2}$ & $W(\phi)$ \\
\hline$-(d / 2)^{2}<m^{2} l^{2}<0$ & $W_{ \pm}(\phi)=-\frac{(d-1)}{\kappa^{2} l}-\frac{1}{2 l} \Delta_{ \pm} \phi^{2}+o\left(\phi^{2}\right)$ \\
\hline$m^{2} l^{2}=-(d / 2)^{2}$ & $W_{+}(\phi)=-\frac{(d-1)}{\kappa^{2} l}-\frac{1}{2 l} \frac{d}{2} \phi^{2}+o\left(\phi^{2}\right)$ \\
& $W_{-}(\phi)=-\frac{(d-1)}{\kappa^{2} l}-\frac{1}{2 l} \frac{d}{2} \phi^{2}\left(1+\frac{1}{\log \phi}\right)+o\left(\frac{\phi^{2}}{\log \phi}\right)$ \\
\hline
\end{tabular}

Table 5: The allowed behavior of the fake superpotential in the vicinity of the AdS critical point at $\phi=0$.

Firstly, from equations (5.2) follows that the asymptotic form of the induced metric and of the scalar field is determined by the form of $W(\phi)$ in the vicinity of $\phi=0$. In particular, requiring the metric to be AlAdS, fixes $W(0)=-(d-1) / \kappa^{2} l$. This, in combination with the form (6.2) of the scalar potential near $\phi=0$, determines, depending on the value of the scalar mass, the allowed behaviors of $W(\phi)$ around $\phi=0$, which are shown in table 5 .

Note that there are two possible asymptotic behaviors in each case. The $W_{+}$solutions imply that the non-normalizable mode, $\phi_{-}(x)$, vanishes in the corresponding solution of the bulk equations of motion, which is obtained from $W(\phi)$ via (5.2). On the other hand, the $W_{-}$solutions allow for a non-zero $\phi_{-}(x)$. Since $\Delta_{ \pm}$are the two roots of the equation $m^{2} l^{2}=\Delta(\Delta-d)$, the requirement that $W(\phi)$ and hence $\Delta_{ \pm}$are real imposes the well known BF bound $m^{2} l^{2} \geq-(d / 2)^{2}$ [22, 27]. Further classification of the possible solutions of equation (6.3) is facilitated by the following lemma.

Lemma 1. Provided the BF bound holds and $\Delta_{-}>0$, any $W_{-}$solution of equation (6.3) lies on a continuous family of $W_{-}$solutions while any $W_{+}$solution is isolated, or corresponds to an end point of an one-parameter family of $W_{-}$solutions, at an infinite distance in parameter space from any given $W_{-}$solution.

To prove this lemma, we will assume that the original solution $W(\phi)$ lies on a oneparameter family of solutions. In the case of $W_{+}$solutions we will show that this always leads to a contradiction, while for $W_{-}$solutions we construct explicitly the one-parameter family of solutions in the neighborhood of $W(\phi)$ when $\Delta_{-}>0$. Suppose then that the solution $W(\phi)$ lies on a continuous family of solutions parameterized by the integration constant $\xi$, chosen such that $\xi=0$ corresponds to $W(\phi)$. The one-parameter family of solutions around $W(\phi)$ then takes the form

$$
W(\phi ; \xi)=W(\phi)+\xi W^{(1)}(\phi)+O\left(\xi^{2}\right)
$$

where

$$
W^{(1)}(\phi)=\exp \left(\frac{d \kappa^{2}}{d-1} \int^{\phi} d \tilde{\phi} \frac{W(\tilde{\phi})}{W^{\prime}(\tilde{\phi})}\right)
$$


Let us now assume that the original solution $W(\phi)$ is of $W_{+}$type. Using the asymptotic form of $W_{+}$solutions for the various masses given in table 5 , we can deduce the corresponding asymptotic behavior of $W^{(1)}(\phi)$. One finds,

$$
W^{(1)}(\phi) \sim\left\{\begin{array}{cc}
\phi^{d / \Delta_{+}}, & -(d / 2)^{2}<m^{2} l^{2}<0, \\
\phi^{2}, & m^{2} l^{2}=-(d / 2)^{2} .
\end{array}\right.
$$

Since $1<d / \Delta_{+}<2$ when $-(d / 2)^{2}<m^{2} l^{2}<0$, we see that in all cases, if one starts with a $W_{+}$solution, the deformed solution $W(\phi ; \xi)$ has asymptotics which are not compatible with the asymptotics in table 5, which any solution must obey. We have therefore reached a contradiction and we conclude that any $W_{+}$type solution is isolated. On the other hand, if the original solution is of $W_{-}$type, then the deformation (6.8) behaves asymptotically as

$$
W^{(1)}(\phi) \sim \begin{cases}\phi^{d / \Delta_{-}}, & -(d / 2)^{2}<m^{2} l^{2}<0, \\ \frac{\phi^{2}}{(\log \phi)^{2}}, & m^{2} l^{2}=-(d / 2)^{2} .\end{cases}
$$

If $\Delta_{-}>0$, the BF bound ensures that $d / \Delta_{-}>2$ and so we see that in this case the asymptotic form of the deformation $W^{(1)}(\phi)$ is subleading relative to the asymptotic behavior of the original solution. Hence, the deformed solution does exist, at least in the neighborhood of the original solution, and it is of $W_{-}$type for any (finite) value of the deformation parameter. ${ }^{18}$ This completes the proof of the above lemma.

The last claim in the the proof of the above lemma, namely that, when the deformation exists, the deformed solution (6.7) remains of $W_{-}$type to all orders in the deformation parameter $\xi$, follows from the next lemma.

Lemma 2. The deformation parameter $\xi$ can be chosen such that all higher-than-first order in $\xi$ terms are also asymptotically subleading relative to $W^{(1)}(\phi)$, i.e. such that $W^{(n)}(\phi)=o\left(W^{(1)}(\phi)\right)$ as $\phi \rightarrow 0$ for all $n>1$.

To prove this statement, we expand the deformed solution $W(\phi ; \xi)$ as

$$
W(\phi ; \xi)=\sum_{n=0}^{\infty} \xi^{n} W^{(n)}(\phi),
$$

where $W^{(0)}(\phi) \equiv W(\phi)$ denotes the undeformed solution. Inserting this in (6.3) we determine that $W^{(1)}(\phi)$ is given by (6.8) while for $n>1$

$$
W^{(n)}(\phi)=W^{(1)}(\phi) \int^{\phi} d \tilde{\phi} \frac{Q^{(n)}}{W^{(0) \prime} W^{(1)}},
$$

where

$$
Q^{(n)} \equiv-\frac{1}{2} \sum_{m=1}^{n-1}\left(W^{(m) \prime} W^{(n-m) \prime}-\frac{d \kappa^{2}}{d-1} W^{(m)} W^{(n-m)}\right)
$$

\footnotetext{
${ }^{18}$ Lemma 2 below guarantees that the asymptotics is not affected by the higher order in $\xi$ terms either.
} 
Using the asymptotics for the $W_{-}$type solutions given in table 5 , we can show that there exists a unique value of the integration constant implicit in (6.12), such that $W^{(2)}(\phi)=$ $o\left(W^{(1)}(\phi)\right)$ as $\phi \rightarrow 0$. Since the integration constant in 6.12 simply multiplies the homogeneous solution $W^{(1)}$, it follows that any other value of the integration constant can be absorbed in the definition of the deformation parameter $\xi$. A simple inductive argument can now be used to complete the proof for any order $n>1$.

Note that in order to determine whether a given $W_{+}$solution is the end point of an oneparameter family of $W_{-}$solutions we need to treat the deformation non-perturbatively, which is to say, we must be able to solve (6.3) exactly. We will consider a case for which this is possible in the next section, where we will show that indeed the $W_{+}$solution is the end point of an one-parameter family of $W_{-}$solutions.

The final ingredient we need to evaluate the renormalized on-shell action for both $W_{+}$ and $W_{-}$type solutions is the asymptotic form of the functions $Z(\phi)$ and $M(\phi)$, given in table 6, which follows from that of the fake superpotential in table 5 via equations (6.4) and (6.5). With this last piece of information then we now only need to insert the functions $W(\phi), Z(\phi)$ and $M(\phi)$ in the regularized action (5.1) and identify the piece of zero dilatation weight. This is straightforward and results in the renormalized actions given in table 7, but a couple of subtle, yet important, points are worth mentioning. Firstly, there is the freedom of adding extra finite local counterterms to $S_{\mathrm{ct}}$, which we mentioned in section 3 . In this case it is manifested by the arbitrariness of the parameter $\xi$ in the effective actions obtained from $W_{-}$solutions. Different $W_{-}$solutions lead to a different value for this parameter and so a definite choice of counterterms amounts to setting this parameter to zero for a particular $W_{-}$solution. Once this choice is made, however, all other $W_{-}$solutions will necessarily have a non-zero $\xi$. It is clear then that for Mixed boundary conditions the freedom of adding finite local counterterms simply corresponds to the choice of what one defines to be the 'undeformed' theory - and not to a renormalization scheme dependence as is the case for Dirichlet boundary conditions. In writing the effective actions in table 0 we have picked a random $W_{-}$solution and we have assigned it the value $\xi=0$. We will see later that if the system (6.1) is embedded in some gauged supergravity, a natural choice for the solution that defines the zero of the parameter $\xi$ is the true superpotential of the theory, provided, of course, it is a $W_{-}$type solution of (6.3). A second minor point to note is that for $d=2$ the leading term of $Z(\phi)$ gives both a divergent term, which is removed by the counterterms, as well as the finite piece that contributes to the renormalized action. This is clear if one splits the logarithm as $\log \phi \sim \log e^{-\Delta_{ \pm} r / l}+\log \phi_{ \pm}(x)$. The first piece then gives the usual logarithmically divergent term associated with the conformal anomaly [44, while the second piece gives the finite contribution to the renormalized action. Note also that we have added an arbitrary function $f\left(\phi_{-}\right)$in the effective actions arising from $W_{-}$ solutions, which corresponds to a general multi-trace deformation. Although the above argument does not account for these terms, we have already seen that they arise from a choice of boundary conditions and we will discuss how they can be accommodated in the the Hamilton-Jacobi setting below. Finally, except from the parameter $\xi$ that appears only 


\begin{tabular}{|l|c|l|l|}
\hline & $m^{2} l^{2}$ & $Z(\phi)$ & $M(\phi)$ \\
\hline$d>2$ & $-(d / 2)^{2}<m^{2} l^{2}$ & $Z_{ \pm}(\phi) \sim-\frac{l}{2 \kappa^{2}(d-2)}+c_{ \pm} \phi^{\frac{d-2}{\Delta \pm}}$ & $M_{ \pm}(\phi) \sim c_{ \pm} \frac{2(d-1)(d-2)}{\Delta_{ \pm}^{2}} \phi^{\frac{d-2}{\Delta \pm}-2}$ \\
\cline { 2 - 4 } & $-(d / 2)^{2}$ & $Z_{+}(\phi) \sim-\frac{l}{2 \kappa^{2}(d-2)}+c_{+} \phi^{\frac{2(d-2)}{d}}$ & $M_{+}(\phi) \sim c_{+} \frac{8(d-1)(d-2)}{d^{2}} \phi^{-\frac{4}{d}}$ \\
\hline$d=2$ & $m^{2}<0$ & $Z_{ \pm}(\phi) \sim \frac{l}{2 \kappa^{2} \Delta_{ \pm}} \log \phi+c_{ \pm}$ & $M_{ \pm}(\phi) \sim \frac{l}{\kappa^{2} \Delta_{ \pm}^{2}} \frac{1}{\phi^{2}}$ \\
\hline
\end{tabular}

Table 6: The asymptotic behavior of the functions $Z(\phi)$ and $M(\phi)$ following from that of the fake superpotential, $W(\phi)$, in table 7.35 via equations $(6.4)$ and $(6.5) \cdot c_{ \pm}$are arbitrary constants corresponding to the integration constant of equation (6.4).

\begin{tabular}{|c|c|c|}
\hline$d$ & $W$ & $\Gamma[\sigma]$ \\
\hline$>2$ & + & $c_{+} \int_{\partial \mathcal{M}} d^{d} x \sqrt{g_{(0)}}\left(\phi_{+}^{\frac{d-2}{\Delta+}} R\left[g_{(0)}\right]+\frac{(d-1)(d-2)}{\Delta_{+}^{2}} \phi_{+}^{\frac{d-2}{\Delta_{+}}-2} g_{(0)}{ }^{i j} \partial_{i} \phi_{+} \partial_{j} \phi_{+}\right)$ \\
& - & $\int_{\partial \mathcal{M}} d^{d} x \sqrt{g_{(0)}}\left(\xi \phi_{-}^{\frac{d}{\Delta_{-}}}+f\left(\phi_{-}\right)+c_{-} \phi_{-}^{\frac{d-2}{\Delta_{-}}} R\left[g_{(0)}\right]+c_{-} \frac{(d-1)(d-2)}{\Delta_{-}^{2}} \phi_{-}^{\frac{d-2}{\Delta_{-}}-2} g_{(0)}{ }^{i j} \partial_{i} \phi_{-} \partial_{j} \phi_{-}\right)$ \\
\hline 2 & + & $\frac{l}{2 \kappa^{2} \Delta_{+}} \int_{\partial \mathcal{M}} d^{d} x \sqrt{g_{(0)}}\left(\log \phi_{+} R\left[g_{(0)}\right]+\frac{2}{\Delta_{+}} \phi_{+}^{-2} g_{(0)}{ }^{i j} \partial_{i} \phi_{+} \partial_{j} \phi_{+}\right)+c_{+} \chi$ \\
& - & $\int_{\partial \mathcal{M}} d^{2} x \sqrt{g_{(0)}}\left(\xi \phi_{-}^{\frac{\Delta_{-}}{\Delta_{-}}}+f\left(\phi_{-}\right)+\frac{l}{2 \kappa^{2} \Delta_{-}}\left(\log \phi_{-} R\left[g_{(0)}\right]+\frac{2}{\Delta_{-}} \phi_{-}^{-2} g_{(0)}{ }^{i j} \partial_{i} \phi_{-} \partial_{j} \phi_{-}\right)\right)+c_{-} \chi$ \\
\hline
\end{tabular}

Table 7: The renormalized effective actions corresponding to the $W_{+}$and $W_{-}$solutions in the two-derivative approximation. $c_{ \pm}$are undetermined constants that depend on the dynamics, while $\chi$ is the Euler number of the two-dimensional boundary. Note that the $d=2$ effective actions are related to the Liouville action by the field redefinition $\varphi=\log \phi_{-}^{1 / \Delta_{-}}$. Interestingly, the parameter $\xi$ corresponds to the 2D cosmological constant.

in the $W_{-}$effective actions, and which as we just saw corresponds to a choice of boundary conditions, the effective actions in table 7 also depend on the undetermined parameters $c_{ \pm}$. These parameters multiply a conformally invariant combination of the two two-derivative terms (for $d>2$ ), and can be determined as we will see by computing the effective potential on a curved boundary, which fixes the coefficient of the curvature term.

The above analysis provides a complete rederivation of the constraints on the scalar mass in order for Dirichlet, Neumann or Mixed boundary to be admissible [22, 34, 35]. However, from a very different perspective. Here the constraints arise as essential conditions for the existence of the corresponding effective action for the dual operator. As we have seen, the existence of this effective action requires first of all the existence of a real function $W(\phi)$, which leads to the BF bound. As expected then, the $W_{+}$solutions, corresponding to Dirichlet boundary conditions, lead to an effective action for the VEV 
$\widehat{\pi}_{\left(\Delta_{+}\right)}=-\frac{1}{l}\left(\Delta_{+}-\Delta_{-}\right) \phi_{+}$, for $\Delta_{+}>\Delta_{-}$, or $\widehat{\pi}_{\left(\Delta_{+}\right)}=-\frac{1}{l} \phi_{+}$, for $\Delta_{+}=\Delta_{-}=d / 2$, of an operator of dimension $\Delta_{+}$. $W_{-}$solutions on the other hand, which correspond to Neumann or Mixed boundary conditions, lead to an effective action for the VEV $\phi_{-}$of an operator of dimension $\Delta_{-}$. It should now be clear why $W_{+}$solutions are isolated while $W_{-}$solutions lie on a one-parameter family of $W_{-}$solutions. Namely, we have seen in section 3 that Dirichlet boundary conditions cannot be continuously deformed, but Neumann and Mixed boundary conditions can. In particular, the parameter $\xi$ that defines this one-parameter family of $W_{-}$solutions is identified with the parameter of a marginal multi-trace deformation. Moreover, we have argued above that these actions should only be valid away from the vanishing VEV point. Indeed, if the kinetic term in the actions in table f could be continued close to zero VEV, this would mean that the two-point function of the scalar operator is dominated by the Goldstone pole in the UV (as well as in the IR), but this of course violates the conformal invariance of the theory which should be restored in the UV. By looking at table 7 we see that the condition for the effective actions to break down for vanishing VEV is precisely the unitarity bound $\Delta_{ \pm}>(d-2) / 2$. Since $\Delta_{+} \geq d / 2$ by definition, this is only a constraint on $\Delta_{-}$, which is equivalent to the condition that the mass lies in the range (3.13). We conclude that Neumann and Mixed boundary conditions, which require the existence of a $W_{-}$solution, are only possible for masses in this range. ${ }^{19}$

\subsection{Minisuperspace approximation}

In order to see how a general multi-trace deformation can be accommodated in the Hamilton-Jacobi language, and possibly to determine the parameters $c_{ \pm}$in the twoderivative effective actions in table 0 by computing the effective potential on a non-flat boundary, we now proceed by considering the 'minisuperspace' approximation for solutions of the form (5.3). From table 10 we see that with this ansatz the canonical momenta reduce to $\pi_{A}=-d(d-1) e^{d A} \dot{A} / \kappa^{2}, \pi_{\phi}=e^{d A} \dot{\phi}$, while the Hamiltonian (A.9) becomes

$$
\mathcal{H}=\frac{1}{2}\left[\left(\pi_{\phi}^{2}-\frac{\kappa^{2}}{d(d-1)} \pi_{A}^{2}\right) e^{-d A}-\left(-\frac{d(d-1) k}{\kappa^{2} l^{2}} e^{-2 A}+2 V(\phi)\right) e^{d A}\right] .
$$

The Hamilton-Jacobi problem then reduces to a standard classical mechanics problem, where we look for a complete integral, $\mathcal{S}(A, \phi)$, such that

$$
\pi_{A}=\frac{\partial \mathcal{S}}{\partial A}, \quad \pi_{\phi}=\frac{\partial \mathcal{S}}{\partial \phi},
$$

and $\mathcal{H}=0$. For $k=0$, i.e. for a flat boundary, a solution to this Hamilton-Jacobi equation is

$$
\mathcal{S}(A, \phi)=e^{d A} W(\phi),
$$

where $W(\phi)$ satisfies equation (6.3). The two equations (6.15) for the momenta then become respectively

$$
\dot{A}=-\frac{\kappa^{2}}{d-1} W(\phi), \quad \dot{\phi}=W^{\prime}(\phi) .
$$

\footnotetext{
${ }^{19}$ Note that this is in complete agreement with the analysis of [21], which shows that a $W_{-}(\phi)$ solution is necessary in order for stability with Mixed boundary conditions to be possible. But from our perspective in terms of the dual field theory, the quantity $W(\phi)$ (called $P(\phi)$ in [21]) is physical and not merely 'an auxiliary construct' - it determines the effective action of the dual operator.
} 
In combination with equation (6.3) for the function $W(\phi)$, we recognize these equations as the flow or 'BPS' equations for Poincaré domain walls (see e.g. 45, 46]). If the action (6.1) is embedded into a particular gauged supergravity, then generically there is a unique solution, $W_{o}(\phi)$, of (6.3) that coincides with the true superpotential of the theory. In that case the flow equations (6.17) do coincide with the true BPS equations of the theory. However, any other solution $W(\phi)$ gives a non-supersymmetric solution of the supergravity equations [47, 23]. Following [48], we call $W(\phi)$ the 'fake superpotential', although 'Hamilton's characteristic function' would be a more appropriate name in the present context. Indeed, none of the above depends on supersymmetry in any way. The first order formalism, also known as 'fake supergravity' 48, for Poincaré domain walls is simply Hamilton-Jacobi theory for the bulk equations of motion [29]. An analysis of curved domain walls, $k \neq 0$, in the context of Hamilton-Jacobi theory has appeared recently in 49. Since the HamiltonJacobi equation arising from the Hamiltonian (6.14) for $k \neq 0$ is non-separable, it is not easy to find a complete integral for an arbitrary potential in this case. Here we will therefore focus on the $k=0$ case, but we will later show that for a conformally coupled scalar such a complete integral can be found even for $k \neq 0$, which will allow us to fix the constants $c_{ \pm}$.

Recall that a complete integral of the Hamilton-Jacobi equation following from the Hamiltonian (6.14) involves an arbitrary constant. In particular, the solution (6.16) is a complete integral provided $W(\phi)$ is the general solution of (6.3), depending on an arbitrary parameter. This parameter, of course, is the coupling, $\xi$, of the marginal multi-trace deformation. Since any complete integral leads to the most general solution of the form (5.3), we can obtain the most general flat domain wall solution provided we can solve equation (6.3) exactly for the one-parameter family of fake superpotentials. Indeed, we will show below that this is possible, at least for certain potentials. However, the complete integral obtained form (6.16) via the one-parameter family of fake superpotentials is not the most general complete integral. To see this, suppose $W_{o}(\phi)$ is a solution of (6.3) such that (6.16) is a solution (not a complete integral) of the Hamilton-Jacobi equation. We can now look at the most general infinitesimal deformation, $\delta \mathcal{S}$, of this solution by linearizing the Hamilton-Jacobi equation around the solution $\mathcal{S}=e^{d A} W_{o}(\phi)$. This gives

$$
W_{o}^{\prime}(\phi) \frac{\partial \delta \mathcal{S}}{\partial \phi}-\frac{\kappa^{2}}{d-1} W_{o}(\phi) \frac{\partial \delta \mathcal{S}}{\partial A}=0
$$

whose general solution is

$$
\delta \mathcal{S}=f\left(e^{A} e^{\left(\frac{\kappa^{2}}{d-1} \int^{\phi} d \bar{\phi} \frac{W_{o}}{W_{o}^{\prime}}\right)}\right)
$$

for an arbitrary function $f$. But note that asymptotically $e^{A} e^{\left(\frac{\kappa^{2}}{d-1} \int^{\phi} d \bar{\phi} \frac{W_{o}}{W_{o}^{\prime}}\right)} \sim \phi_{ \pm}^{1 / \Delta_{ \pm}}$, depending on whether $W_{o}$ is a $W_{+}$or $W_{-}$solution. It follows that $\delta \mathcal{S}$ contributes to the renormalized action and corresponds to an arbitrary multi-trace deformation. However, if this deformation were allowed for both $W_{+}$and $W_{-}$solutions, it would contradict our previous conclusion that multi-trace deformations are allowed only for Neumann or Mixed 
boundary conditions, and hence, only for $W_{-}$solutions. Indeed, under the deformation $\delta \mathcal{S}$,

$$
\delta \dot{\phi} \sim \frac{1}{\Delta_{ \pm}} f^{\prime}\left(e^{A} \phi^{1 / \Delta_{ \pm}}\right) e^{-(d-1) A} \phi^{\left(1-\Delta_{ \pm}\right) / \Delta_{ \pm}} .
$$

In order for the asymptotic form of the scalar field, $\phi \sim \phi_{ \pm}(x) e^{-\Delta_{ \pm}}$, not to be changed by the deformation, we must then require that $d-1+1-\Delta_{ \pm} \geq \Delta_{ \pm}$, or equivalently $\Delta_{ \pm} \leq d / 2$. But this picks out only $\Delta_{-}$since by definition $\Delta_{+} \geq d / 2$. We therefore conclude that the deformation (6.19) is allowed only if $W_{o}$ is a $W_{-}$solution.

The above discussion demonstrates that in the Hamilton-Jacobi formalism, any multitrace deformation corresponds to a choice of a complete integral of the Hamilton-Jacobi equation. In particular, although a complete integral of the form (6.16) accounts only for marginal multi-trace deformations, by allowing for a more general complete integral as in (6.19) the Hamilton-Jacobi formalism can accommodate any multi-trace deformation. This freedom in choosing a complete integral then gives rise to the arbitrary function $f\left(\phi_{-}\right)$ in the corresponding renormalized effective action.

\subsection{The ' $2 / 3$ ' potential}

We now consider a special scalar potential for which equation (6.3) for the fake superpotential can be solved exactly. As we have seen, this gives a complete integral of the Hamilton-Jacobi equation via (6.16), and hence the most general flat domain wall solution. The potential we will consider is

$$
V(\phi)=-\frac{d(d-1)}{2 \kappa^{2} l^{2}} \cosh \left(\frac{2}{3} \sqrt{\frac{d \kappa^{2}}{d-1}} \phi\right),
$$

which we propose to call the ' $2 / 3$ ' potential. This potential was introduced in [32], although the special case $d=3$ has appeared elsewhere in the literature as well. In particular, for $d=3$ this potential arises from a one-scalar consistent truncation of the $\mathcal{N}=8$ gauged supergravity in $D=d+1=4$ dimensions [50. It was also considered in 51, where a four-dimensional asymptotically locally AdS topological black hole with scalar hair was found, as well as in [23] and [24], where respectively four-dimensional domain walls and instantons were found and uplifted to M-theory.

The scalar mass for the potential (6.21) is $m^{2} l^{2}=-2(d / 3)^{2}$ and hence the two conformal dimensions are $\Delta_{-}=d / 3, \Delta_{+}=2 d / 3$. Requiring that the mass falls in the range (3.13), for which Mixed boundary conditions can be considered, restricts the boundary dimension to lie in the range $2 \leq d \leq 6$. In [32] it was shown that equation (6.3) with the potential (6.21) can be solved exactly. The general solution is (see also 23])

$$
W(\phi ; \nu)=-\frac{d-1}{\kappa^{2} l} \frac{1}{\left(1-\rho^{2}\right)^{\frac{3}{4}}} \frac{1-\rho^{2}+\sqrt{1+2 \nu \rho+\rho^{2}}}{\sqrt{2\left(1+\nu \rho+\sqrt{1+2 \nu \rho+\rho^{2}}\right)}}, \quad \nu \geq-1,
$$

where $\rho=\tanh \left(\frac{2}{3} \sqrt{\frac{d \kappa^{2}}{d-1}} \phi\right)$ and $\nu \geq-1$ is an arbitrary parameter. In the $d=3$ case, the value $\nu=-1$ corresponds to the true superpotential of the truncated $\mathcal{N}=8$ gauged 
supergravity in four dimensions [23]. Expanding (6.22) one obtains

$$
W(\phi ; \nu)=-\frac{d-1}{\kappa^{2} l}\left(1+\frac{1}{6} \psi^{2}+\frac{1}{27} \nu \psi^{3}+\mathcal{O}\left(\psi^{4}\right)\right),
$$

where $\psi=\sqrt{\frac{d \kappa^{2}}{d-1}} \phi$. For any finite $\nu \geq-1$ then (6.22) is a $W_{-}$type solution and the parameter $\nu$ is related to the deformation parameter $\xi$ in (6.7). In particular, choosing $\xi$ such that $\xi=0$ corresponds to the supersymmetric solution with $\nu=-1$ gives $\xi=$ $-\frac{d-1}{27 \kappa^{2} l}\left(\frac{d \kappa^{2}}{d-1}\right)^{\frac{3}{2}}(\nu+1)$.

The flow equations (6.17) can now be used to construct the corresponding Poincaré domain wall solution, which takes the form

$$
\begin{aligned}
d s^{2}= & \left(\frac{3 l}{d}\right)^{2} \frac{\left(1+\nu \rho+\sqrt{1+2 \nu \rho+\rho^{2}}\right)}{2 \rho^{2} \sqrt{1-\rho^{2}}\left(1+2 \nu \rho+\rho^{2}\right)} d \rho^{2} \\
& +c^{2}\left(\frac{\sqrt{1-\rho^{2}}}{2 \rho^{2}}\left(1+\nu \rho+\sqrt{1+2 \nu \rho+\rho^{2}}\right)\right)^{3 / d} d x^{i} d x^{i}, \\
\phi= & \frac{3}{2} \sqrt{\frac{d-1}{d \kappa^{2}}} \tanh ^{-1} \rho,
\end{aligned}
$$

where, for finite $\nu$, the parameter $c$ is related to the $\mathrm{VEV}, \phi_{-}$, via

$$
c=\left(\frac{2}{3} \sqrt{\frac{d \kappa^{2}}{d-1}} \phi_{-}\right)^{3 / d} .
$$

For the special case $d=3$, where this domain wall is a solution of $\mathcal{N}=8$ gauged supergravity, the value $\nu=-1$ gives a supersymmetric domain wall since for this value (6.22) coincides with the true superpotential. For $\nu>-1(6.24)$ is a non-supersymmetric solution of the equations of motion.

We have seen that every solution of the bulk equations of motion is dual to an extremum, or 'vacuum', of the effective action of the dual boundary theory. In particular, Poincaré domain walls correspond to homogeneous vacua, where the VEV $\phi_{-}$is a constant extremizing the effective potential $V_{\text {eff }}\left(\phi_{-}\right)$. From our general prescription for computing the effective action in section 3, we see that in order to evaluate the effective potential we should first evaluate the renormalized momentum as a functional of the VEV by solving the radial problem. We have already done this in this case and we have found $\widehat{\pi}_{\left(\Delta_{+}\right)}=d \xi \phi_{-}^{\Delta_{+} / \Delta_{-}} / \Delta_{-}=3 \xi \phi_{-}^{2}$. However, this depends on the arbitrary parameter $\xi$, which should in principle be fixed by requiring regularity of the corresponding solution (6.24). But since this solution is singular for any value of $\xi{ }^{20}$ we exceptionally do not impose this condition and we will take $\xi \leq 0$ to be arbitrary. The effective potential then takes the form

$$
V_{\text {eff }}\left(\phi_{-}\right)=\xi \phi_{-}^{3}+f\left(\phi_{-}\right),
$$

\footnotetext{
${ }^{20}$ Note, however that while for $\nu=-1$ 6.24 has a null singularity, for $\nu>-1$ the singularity is timelike 23]. If this is taken as a criterion for 'regularity', then the supersymmetric solution is the only 'regular' solution.
} 
where $\xi$ is related to $\nu$ by the relation we gave above. An extremum of this effective potential then corresponds to imposing the relation

$$
3 \xi \phi_{-}^{2}+f^{\prime}\left(\phi_{-}\right)=0,
$$

between the parameters $\nu$ and $\phi_{-}$. The domain wall (6.24) with this relation imposed is then dual to the vacuum of the boundary theory corresponding to the VEV given by (6.27). We should emphasize here that given a solution of (6.27), the corresponding domain wall (6.24) takes the same form for all choices of boundary condition $f\left(\phi_{-}\right)$, although the relation between $\nu$ and $\phi_{-}$is different for different boundary conditions.

An interesting example is the case where the domain walls $(\sqrt[6.24]{ })$ are solutions of $\mathcal{N}=8$ gauged supergravity in four dimensions. In that case, the $\nu=-1(\xi=0)$ domain wall is supersymmetric and describes the Coulomb branch of the dual theory. The corresponding effective potential is therefore flat, i.e. $f\left(\phi_{-}\right) \equiv 0$, since the VEV is totally arbitrary. If the coupling $\xi$ of the marginal multi-trace deformation is then turned on, the effective potential, $V_{\text {eff }}\left(\phi_{-}\right)=\xi \phi_{-}^{3}$, destabilizes the theory since $\xi<0 .{ }^{21}$ To have a non-trivial solution when $\nu>-1$ then, we need to introduce another $\xi$-dependent deformation, on top of the marginal one. In particular, we can choose $f\left(\phi_{-}\right)=-\xi h\left(\phi_{-}\right)$such that the effective potential is

$$
V_{\text {eff }}\left(\phi_{-}\right)=\xi\left(\phi_{-}^{3}-h\left(\phi_{-}\right)\right)
$$

for some function $h\left(\phi_{-}\right)>0$. Even though for $\xi=0$ the VEV is totally undetermined, when $\xi$ is turned on the VEV is fixed to some non-zero value determined by $V_{\text {eff }}^{\prime}\left(\phi_{-}\right)=0$. This is precisely the situation discussed in [23], where the specific choice $h\left(\phi_{-}\right)=J_{*} \phi_{-}$, corresponding to a single-trace deformation, was made. While at the supersymmetric point describing the Coulomb branch the VEV is arbitrary, away from the supersymmetric point the VEV is a function of the arbitrary background source, $J_{*}$, of the single-trace deformation.

Finally, let us consider the limit $\nu \rightarrow \infty$, corresponding to the limit where the coupling, $\xi$, of the marginal deformation of the original CFT is sent to (negative) infinity. In this limit the fake superpotential (6.22) becomes

$$
W(\phi ; \infty)=-\frac{d-1}{\kappa^{2} l} \frac{1}{\left(1-\rho^{2}\right)^{\frac{3}{4}}} .
$$

Expanding this we get

$$
W(\phi ; \infty)=-\frac{d-1}{\kappa^{2} l}\left(1+\frac{1}{3} \psi^{2}+\mathcal{O}\left(\psi^{4}\right)\right)
$$

and so this is a $W_{+}$solution. The one-parameter family of fake superpotentials (6.22) then provides an explicit example of the general picture we discussed in the previous section. Namely, for all finite values of $\nu, 6$ 6.22) is a $W_{-}$solution, while the $W_{+}$solution arises as

\footnotetext{
${ }^{21}$ If $\xi$ were positive, the effective potential would force the VEV to vanish. In that case the domain wall (6.24) reduces to exact AdS. A non-trivial solution for $\nu>-1$ then would require the addition of some other term proportional to $\xi$ in the effective potential, much like in the case $\xi<0$.
} 
an endpoint of this one-parameter family at $\nu \rightarrow \infty$. Letting $c^{2} \nu^{\frac{3}{d}}=\bar{c}^{2}$, the domain wall solution corresponding to $\nu \rightarrow \infty$ is

$$
\begin{aligned}
d s^{2} & =\left(\frac{3 l}{d}\right)^{2} \frac{d \rho^{2}}{4 \rho^{2} \sqrt{1-\rho^{2}}}+\bar{c}^{2}\left(\frac{\sqrt{1-\rho^{2}}}{2 \rho}\right)^{3 / d} d x^{i} d x^{i}, \\
\phi & =\frac{3}{2} \sqrt{\frac{d-1}{d \kappa^{2}}} \tanh ^{-1} \rho,
\end{aligned}
$$

where now

$$
\bar{c}=\left(\frac{4}{3} \sqrt{\frac{d \kappa^{2}}{d-1}} \phi_{+}\right)^{3 / 2 d} .
$$

For an infinite value of the marginal deformation parameter then the dimension of the operator dual to the scalar field changes from $\Delta_{-}$to $\Delta_{+}$. The domain wall (6.31) describes the arbitrary VEV of this dimension $\Delta_{+}$operator.

\section{Conformal coupling}

As a second example of a system where the method outlined in section 5 can be applied, we consider the minimally coupled scalar field in (6.1) with the strange-looking potential

$$
\begin{aligned}
V(\phi)= & -\frac{d(d-1)}{2 \kappa^{2} l^{2}}\left(\cosh \left(\sqrt{\frac{(d-1) \kappa^{2}}{4 d}} \phi\right)\right)^{\frac{2(d+1)}{(d-1)}} \\
& +\frac{\lambda}{2}\left(\frac{4 d}{(d-1) \kappa^{2}}\right)^{\frac{(d+1)}{(d-1)}}\left(\sinh \left(\sqrt{\frac{(d-1) \kappa^{2}}{4 d}} \phi\right)\right)^{\frac{2(d+1)}{(d-1)}}
\end{aligned}
$$

where $\lambda$ is an arbitrary dimensionless coupling constant, which we will assume it is positive. Although this potential looks rather complicated and unintuitive, it is in fact a very special potential. First, note that the scalar mass corresponding to the potential (7.1) is the conformal mass $m^{2} l^{2}=-(d / 2)^{2}+1 / 4$, leading to the two conformal dimensions $\Delta_{ \pm}=$ $(d \pm 1) / 2$. Scalars with this mass in AdS are 'massless' in the sense that their Lorentzian bulk-to-bulk propagator has support only on the light cone $d\left(x, x^{\prime}\right)=0$, where $d\left(x, x^{\prime}\right)$ is the geodesic distance between two points $x$ and $x^{\prime}$ in AdS [22]. Moreover, the conformal mass falls within the mass range (3.13) which allows for Mixed boundary conditions.

However, the conformal mass is not the only special property of the potential (7.1). Another special property of the potential (7.1) is that for $d=3$ and $\lambda=\kappa^{2} / 6 l^{2}$ it coincides with the potential (6.21), which, as we pointed out, precisely for $d=3$ can be embedded into $\mathcal{N}=8$ gauged supergravity in four dimensions. The most significant property though of (7.1) is that the field redefinition

$$
\begin{aligned}
\sqrt{(d-1) / d} \kappa \tilde{\phi} / 2 & =\tanh (\sqrt{(d-1) / d} \kappa \phi / 2) \\
\tilde{g}_{\mu \nu} & =(\cosh (\sqrt{(d-1) / d} \kappa \phi / 2))^{\frac{4}{(d-1)}} g_{\mu \nu}
\end{aligned}
$$


transforms the action (6.1) with the potential (7.1) into the form ${ }^{22}$

$$
S=\int_{\mathcal{M}} d^{d+1} x \sqrt{g}\left(-\frac{1}{2 \kappa^{2}}\left(R+\frac{d(d-1)}{l^{2}}\right)+\frac{1}{2} g^{\mu \nu} \partial_{\mu} \phi \partial_{\nu} \phi+\frac{d-1}{8 d} R \phi^{2}+\frac{\lambda}{2} \phi^{\frac{2(d+1)}{(d-1)}}\right),
$$

which is the action for a self-interacting scalar conformally coupled to $\mathrm{AdS}_{d+1}$ gravity. This transformation was given for the cases $d=2$ and $d=3$ in [11] and [51 respectively. This last property allows us to circumvent the problem of analyzing the action (6.1) with the complicated potential (7.1), by studying instead the equivalent but simpler action (7.3). Note, however, that the transformation (7.2) implies that the conformally coupled scalar can be transformed into a minimally coupled scalar provided it is bounded. Since the action (7.3) does not necessarily imply that the scalar field is bounded, only certain bounded solutions of (7.3) correspond to solutions of (6.1).

The equations of motion following from the action (7.3) can be written in the form

$$
R_{\mu \nu}+\frac{d}{l^{2}} g_{\mu \nu}=\kappa^{2} \mathcal{T}_{\mu \nu}, \quad \square_{g} \phi-\frac{d-1}{4 d} R \phi-\frac{d+1}{d-1} \lambda \phi^{(d+3) /(d-1)}=0,
$$

where the modified stress tensor $\mathcal{T}_{\mu \nu}$ is given by

$$
\mathcal{T}_{\mu \nu}=\frac{(d-1)^{2}}{4 d} \frac{\phi^{2 d /(d-1)}}{\left(1-\frac{(d-1) \kappa^{2}}{4 d} \phi^{2}\right)}\left(\nabla_{\mu} \nabla_{\nu}-\frac{1}{d+1} g_{\mu \nu} \square_{g}\right) \phi^{-2 /(d-1)} .
$$

These equations are in fact not independent. Since $\mathcal{T}_{\mu \nu}$ is manifestly traceless, the first equation in (7.4) implies that the Ricci scalar is constant

$$
R=-\frac{d(d+1)}{l^{2}}
$$

The contracted Bianchi identity then implies that $\mathcal{T}_{\mu \nu}$ is divergenceless. This fact imposes a differential constraint on the scalar $\phi$ which is precisely the second equation in (7.4), except that the dimensionless coupling appears as an integration constant and so is not determined by the divergencelessness of $\mathcal{T}_{\mu \nu}$. Hence, the first equation in (7.4) implies the second up to the value of the dimensionless coupling.

The very special form of these equations of motion makes it much easier to study the action (7.3) instead of the minimally coupled scalar described by the action (6.1) with the potential (6.21)- or any other potential, in fact. However, the conformal coupling in the action (7.3) requires that we revisit not only the variational problem, but also the derivation of the holographic effective action. In appendix $\mathrm{A}$ we consider the variational problem for both the actions (6.1) and (7.3) in detail, and in each case we derive the correct form of the Gibbons-Hawking term, as well as the radial canonical momenta, both of which are listed in table 10. Using these results we can now turn to the computation of the effective action for the operator dual to the scalar field described by the action (7.3).

\footnotetext{
${ }^{22}$ Note that we have dropped the tildes from the action $(7.3)$ to simplify the formulas that follow. It should be clear from the context when $\phi$ denotes the minimally coupled scalar in (6.1) or the conformally coupled scalar in $(7.3)$.
} 


\subsection{Two-derivative effective action for conformal boundary conditions}

To compute the renormalized effective action in the two-derivative approximation we proceed as in the case of minimal coupling. Namely, one inserts the ansatz (5.1) for the regularized action into the momentum and Hamiltonian constraints (A.8). The momentum constraint is independent of the particular form of the canonical momenta and, as in the case of minimal coupling, it is automatically satisfied since it simply reflects the invariance of (5.1) with respect to $\Sigma_{r}$ diffeomorphisms. Since the Hamiltonian (A.10) is now different, however, the Hamiltonian constraint leads to the equations

$$
\begin{gathered}
W^{\prime 2}-\frac{\kappa^{2}}{d(d-1)}\left(d W-\frac{d-1}{2} \phi W^{\prime}\right)^{2}=-\frac{d(d-1)}{\kappa^{2} l^{2}}+\lambda \phi^{\frac{2(d+1)}{d-1}} \\
{\left[W^{\prime}+\frac{\kappa^{2}}{2 d} \phi\left(d W-\frac{d-1}{2} \phi W^{\prime}\right)\right] Z^{\prime}-\frac{\kappa^{2}}{d}\left(\frac{d-2}{d-1}\right) \times} \\
\times\left(d W-\frac{d-1}{2} \phi W^{\prime}\right) Z+\frac{1}{2 \kappa^{2}}\left(1-\frac{(d-1) \kappa^{2}}{4 d} \phi^{2}\right)=0 \\
M=\frac{\frac{2 \kappa^{2}}{d}\left(d W-\frac{d-1}{2} \phi W^{\prime}\right) Z^{\prime}+\frac{d-1}{2 d} \phi}{W^{\prime}+\frac{\kappa^{2}}{2 d} \phi^{2}\left(d W-\frac{d-1}{2} \phi W^{\prime}\right)}
\end{gathered}
$$

instead of equations (6.3), (6.4) and (6.5). The action is therefore determined once we solve the non-linear equation (7.7), which is the analogue of (6.3) for minimal coupling.

The general solution of (7.7) in the vicinity of the exact solution corresponding to $\xi=0$ takes the form

$$
W(\phi ; \xi)=-\frac{d-1}{\kappa^{2} l}+\left( \pm \frac{d-1}{2 d} \sqrt{\lambda}+\xi\left(1 \mp \frac{2 l \sqrt{\lambda}}{d-1} \phi^{\frac{2}{d-1}}\right)^{-d}\right) \phi^{\frac{2 d}{d-1}}+\mathcal{O}\left(\xi^{2}\right),
$$

where $\xi$ is an arbitrary parameter analogous to the deformation parameter in (6.8). Note that both sign possibilities here lead to solutions analogous to the $W_{-}$solutions of equation $(6.3)$ (i.e. $\phi_{-} \neq 0$ ). Perhaps the analogue of a $W_{+}$solution can be obtained in the limit $\xi \rightarrow \infty$, which could be evaluated if the solution (7.10) were known exactly as a function of $\xi$, but we will not investigate this further. We will fix the sign in (7.10) below. Using $(7.10)$ we find that the leading asymptotic behavior of the functions $Z(\phi)$ and $M(\phi)$ is exactly as given in table 6 for a $W_{-}$solution with $\Delta_{-}=(d-1) / 2$. Evaluating then the renormalized action we obtain

$$
\Gamma\left[\phi_{-}\right]=\left\{\begin{array}{c}
\int_{\partial \mathcal{M}} d^{d} x \sqrt{g_{(0)}}\left(V_{\text {eff }}\left(\phi_{-}\right)+c_{-}\left(\phi_{-}^{\frac{2(d-2)}{(d-1)}} R\left[g_{(0)}\right]+\frac{4(d-2)}{(d-1)} \phi_{-}^{-\frac{2}{(d-1)}} g_{(0)}{ }^{i j} \partial_{i} \phi_{-} \partial_{j} \phi_{-}\right)\right), \\
\int_{\partial \mathcal{M}} d^{2} x \sqrt{g_{(0)}}\left(V_{\text {eff }}\left(\phi_{-}\right)+\frac{l}{\kappa^{2}}\left(\log \phi_{-} R\left[g_{(0)}\right]+4 \phi_{-}^{-2} g_{(0)}{ }^{i j} \partial_{i} \phi_{-} \partial_{j} \phi_{-}\right)\right)+c_{-} \chi, \\
d=2,
\end{array}\right.
$$

which, as expected, are identical with the renormalized actions in table 0, except that the effective potential is now given by

$$
V_{\text {eff }}\left(\phi_{-}\right)=\left( \pm \frac{d-1}{2 d} \sqrt{\lambda}+\xi\right) \phi_{-}^{\frac{2 d}{d-1}}+f\left(\phi_{-}\right) .
$$




\subsection{Minisuperspace approximation}

As for the minimally coupled scalar, the next step is to look at the 'minisuperspace' of solutions of the form (5.3). The canonical momenta dual to the warp factor, $A(r)$, and the scalar field, which can be deduced from the momenta given in table 10, are respectively

$$
\begin{aligned}
& \pi_{A}=e^{d A}\left(-\frac{d(d-1)}{\kappa^{2}}\left(1-\frac{(d-1) \kappa^{2}}{4 d} \phi^{2}\right) \dot{A}+\frac{d-1}{2} \phi \dot{\phi}\right), \\
& \pi_{\phi}=e^{d A}\left(\dot{\phi}+\frac{d-1}{2} \dot{A} \phi\right) .
\end{aligned}
$$

Moreover, the Hamiltonian (A.10) becomes

$$
\begin{aligned}
\mathcal{H}=\frac{1}{2}\left\{e^{-d A}(\right. & \left.\pi_{\phi}^{2}-\frac{\kappa^{2}}{d(d-1)}\left(\pi_{A}-\frac{d-1}{2} \phi \pi_{\phi}\right)^{2}\right) \\
& \left.+e^{d A}\left(\frac{d(d-1)}{\kappa^{2} l^{2}}-\lambda \phi^{\frac{2(d+1)}{(d-1)}}+\frac{d(d-1) k}{\kappa^{2} l^{2}} e^{-2 A}\left(1-\frac{(d-1) \kappa^{2}}{4 d} \phi^{2}\right)\right)\right\} .
\end{aligned}
$$

Writing again

$$
\pi_{A}=\frac{\partial \mathcal{S}}{\partial A}, \quad \pi_{\phi}=\frac{\partial \mathcal{S}}{\partial \phi},
$$

and inserting these into the equation $\mathcal{H}=0$ for the Hamiltonian (7.14) we obtain the Hamilton-Jacobi equation for the conformally coupled scalar.

We could now look for a solution of the form (6.16), in which case the HamiltonJacobi equation requires that the fake superpotential satisfies equation (7.7). Indeed, the exact solution obtained from (7.10) by setting $\xi=0$ does give a solution to the HamiltonJacobi equation. However, since we do not know the full one-parameter family of fake superpotentials that solve (7.7), the corresponding solution of the Hamilton-Jacobi equation is not a complete integral, which is necessary in order to obtain the most general domain wall solutions of the equations of motion. Nevertheless, in this case we can find a complete integral of the Hamilton-Jacobi equation that is not of the form (6.16) and it is valid even for curved boundary, $k= \pm 1$, as well as for flat boundary. It is easy to verify that writing

$$
\begin{aligned}
& \pi_{A}=-\frac{d(d-1)}{\kappa^{2} l} e^{d A} \sqrt{1+k e^{-2 A}+\mu e^{-(d+1) A}}+\frac{d-1}{2} \phi \pi_{\phi}, \\
& \pi_{\phi}= \pm e^{d A} \sqrt{\frac{d(d-1)}{\kappa^{2} l^{2}} \mu e^{-(d+1) A}+k\left(\frac{d-1}{2 l}\right)^{2} \phi^{2} e^{-2 A}+\lambda \phi^{\frac{2(d+1)}{(d-1)}}}
\end{aligned}
$$

where $\mu$ is an arbitrary parameter, in the Hamiltonian (7.14) automatically gives $\mathcal{H}=0$. Of course, this does not mean that we have found a solution to the Hamilton-Jacobi equation unless

$$
\frac{\partial \pi_{A}}{\partial \phi}=\frac{\partial \pi_{\phi}}{\partial A} .
$$

Remarkably, this is indeed the case and hence there exists a complete integral $\mathcal{S}(A, \phi)$ such that the momenta (7.16) are obtained from it via $(\overline{7.15})$. The fact that the momenta $(7.16)$ 
are integrable, i.e. that they can be derived from a complete integral $\mathcal{S}(A, \phi)$ via (7.15), is one of our main results. As we will now show, this complete integral of the HamiltonJacobi equation will allow us not only to completely determine the two-derivative effective action on any boundary of locally constant scalar curvature, but also to obtain all possible solutions of the form (5.3).

Effective action. Expanding the momenta (7.16) in eigenfunctions of the dilatation operator and keeping the term of weight zero gives immediately the renormalized momenta

$$
\begin{aligned}
\widehat{\pi}_{(d) j}^{i} & =\frac{1}{2}\left(1+(-1)^{d}\right) \frac{(-1)^{\frac{d}{2}} k^{\frac{d}{2}} \Gamma(d+1)}{2^{d+1} l \kappa^{2}\left(\Gamma\left(\frac{d}{2}+1\right)\right)^{2}} \delta_{j}^{i}+\frac{d-1}{4 d} \phi_{-} \widehat{\pi}_{\left(\Delta_{+}\right)} \delta_{j}^{i}, \\
\widehat{\pi}_{\left(\Delta_{+}\right)} & = \pm \sqrt{\frac{d(d-1)}{\kappa^{2} l^{2}} \mu+k\left(\frac{d-1}{2 l}\right)^{2} \phi_{-}^{2}+\lambda \phi_{-}^{\frac{2(d+1)}{(d-1)}}}
\end{aligned}
$$

where we have traded again the momentum $\pi_{A}$ for the physical momentum conjugate to the induced metric $\gamma_{i j}$. Note that the first term in $\widehat{\pi}_{(d) j}^{i}$, which only appears for even boundary dimension, is nothing but the conformal anomaly [44], as can be seen from the relation between the VEV of the stress tensor and the renormalized momentum $\widehat{\pi}_{(d) j}^{i}$ given in table 3 .

The first thing that these renormalized momenta can tell us is the value of the undetermined parameter $c_{-}$in the effective action (7.11). Note that the parameter $\mu$ is determined as a function of the VEV $\phi_{-}$by the requirement of regularity for the corresponding domain wall, which we will discuss below. As we will show, a possible value is $\mu=0$ - in fact the only possible value for the physically relevant case where $(7.3)$ is embedded in $\mathcal{N}=8$ gauged supergravity in four dimensions. Choosing $\mu=0$ then and expanding $\widehat{\pi}_{\left(\Delta_{+}\right)}$for small curvature (large $l$ ), we obtain

$$
\widehat{\pi}_{\left(\Delta_{+}\right)}= \pm \sqrt{\lambda} \phi_{-}^{\frac{d+1}{d-1}}\left(1+\frac{k}{2 \lambda}\left(\frac{d-1}{2 l}\right)^{2} \phi_{-}^{-\frac{4}{d-1}}+\cdots\right)
$$

where the dots stand for higher derivative terms. Comparing this with the derivative of the effective action (7.11) with respect to the VEV, $\phi_{-}$, and using $R\left[g_{(0)}\right]=d(d-1) / l^{2}$, determines

$$
c_{-}= \pm \frac{(d-1)^{2}}{16 d(d-2) \sqrt{\lambda}}, \quad d>2, \quad \sqrt{\lambda}=\kappa^{2} / 16 l, \quad d=2 .
$$

Note in particular that for $d=2$ the coupling $\lambda$ is itself fixed. In order to have a positive kinetic term in the effective action (7.11), we should choose the positive sign in the renormalized momentum $\widehat{\pi}_{\left(\Delta_{+}\right)}$and hence in the momentum (7.16).

However, the renormalized momentum $\widehat{\pi}_{\left(\Delta_{+}\right)}$in (7.18) allows us to determine the exact effective potential on any boundary of constant scalar curvature. Namely, integrating $\widehat{\pi}_{\left(\Delta_{+}\right)}$ 


\begin{tabular}{|c|c|}
\hline$d$ & $V_{k=1}\left(\phi_{-}\right)$ \\
\hline 2 & $\frac{1}{8 l}\left(\phi_{-}^{2} \sqrt{1+4 l^{2} \lambda \phi_{-}^{4}}+\frac{1}{2 l \sqrt{\lambda}} \log \left(2 l \sqrt{\lambda} \phi_{-}^{2}+\sqrt{1+4 l^{2} \lambda \phi_{-}^{4}}\right)\right)+f\left(\phi_{-}\right)$ \\
3 & $\frac{1}{3 l^{3} \lambda}\left(\left(1+l^{2} \lambda \phi_{-}^{2}\right)^{3 / 2}-1\right)+f\left(\phi_{-}\right)$ \\
4 & $\frac{3}{8 \lambda}\left(\frac{3}{2 l}\right)^{3}\left(\phi_{-}^{2 / 3}\left(\frac{1}{2}+\frac{4 l^{2}}{9} \lambda \phi_{-}^{4 / 3}\right) \sqrt{1+\frac{4 l^{2}}{9} \lambda \phi_{-}^{4 / 3}}-\frac{1}{4 l \sqrt{\lambda}} \log \left(\frac{2 l}{3} \phi_{-}^{2 / 3}+\sqrt{1+\frac{4 l^{2}}{9} \lambda \phi_{-}^{4 / 3}}\right)\right)+f\left(\phi_{-}\right)$ \\
\hline
\end{tabular}

Table 8: The exact effective potential (7.21) for $k=1$ and $d=2,3,4$. Note that for $d=2$ the coupling, $\lambda$, is given by $(7.20)$.

with respect to $\phi_{-}$(for $\mu=0$ again) we obtain the exact effective potential

$$
\begin{aligned}
V_{k}\left(\phi_{-}\right)= & \frac{(d-1)^{3} k}{8 d(d-2) l^{2} \sqrt{\lambda}} \phi_{-}^{\frac{2(d-2)}{(d-1)}} F\left(1-\frac{d}{2}, \frac{1}{2} ; 2-\frac{d}{2} ;-\frac{k}{\lambda}\left(\frac{d-1}{2 l}\right)^{2} \phi_{-}^{-\frac{4}{(d-1)}}\right) \\
& +\frac{(d-1)}{2 d} \phi_{-} \sqrt{\left(\frac{d-1}{2 l}\right)^{2} k \phi_{-}^{2}+\lambda \phi_{-}^{\frac{2(d+1)}{(d-1)}}}+V_{o}+f\left(\phi_{-}\right),
\end{aligned}
$$

where the overall constant

$$
V_{o}=-\frac{\Gamma\left(2-\frac{d}{2}\right) \Gamma\left(\frac{d-1}{2}\right)}{\Gamma\left(\frac{1}{2}\right)} \frac{(d-1)^{d+1} k^{\frac{d}{2}}}{2^{d+1} d(d-2) l^{d} \lambda^{\frac{d-1}{2}}},
$$

is determined by the requirement that $V_{k}(0)=0$. For $k=0$ the effective potential (7.21) reduces to

$$
V_{k=0}\left(\phi_{-}\right)=\frac{(d-1)}{2 d} \sqrt{\lambda} \phi_{-}^{\frac{2 d}{(d-1)}}+f\left(\phi_{-}\right) .
$$

For $k=1$ and for $d=2,3,4$ the potential (7.21) is explicitly shown in table 8 .

Domain walls and (absence of) gravitational instantons. The solution (7.16) also enables us to find all possible solutions of the form (5.3). Using the expressions (7.13) for the canonical momenta in terms of the radial derivatives of the warp factor and of the scalar field we find

$$
d r=\frac{l d A}{\sqrt{1+k e^{-2 A}+\mu e^{-(d+1) A}}}
$$

where $\mu$ is an arbitrary integration constant. Defining $u \equiv e^{-A}$ and $\varphi \equiv u^{-(d-1) / 2} \phi$ we can then write down the most general domain wall $(5.3)$ in the form

$$
\begin{gathered}
d s^{2}=\frac{l^{2} d u^{2}}{u^{2}\left(1+k u^{2}+\mu u^{d+1}\right)}+\frac{1}{u^{2}} d s_{d}^{2} . \\
\int_{\varphi_{o}}^{\varphi} \frac{d \bar{\varphi}}{\sqrt{\frac{d(d-1)}{\kappa^{2} l^{2}} \mu+k \frac{(d-1)^{2}}{4 l^{2}} \bar{\varphi}^{2}+\lambda \bar{\varphi}^{\frac{2(d+1)}{(d-1)}}}}=\mp l \int_{0}^{u} \frac{d \bar{u}}{\sqrt{1+k \bar{u}^{2}+\mu \bar{u}^{d+1}}} .
\end{gathered}
$$




\begin{tabular}{|l|l|l|}
\hline$k$ & $d s_{d}^{2}$ & \\
\hline 0 & $d s^{2}\left(\mathbb{R}^{d}\right)=d x^{i} d x^{i}$ & $z=u, z^{i}=x^{i}$ \\
1 & $d s^{2}\left(S^{d}\right)=d \theta^{2}+\sin ^{2} \theta d s^{2}\left(S^{d-1}\right)$ & $z=\frac{u}{\sqrt{1+u^{2}}+\cos \theta}, z^{i}=\frac{\sin \theta n^{i}}{\sqrt{1+u^{2}}+\cos \theta}, n^{i} n^{i}=1$ \\
-1 & $d s^{2}\left(\mathbb{H}_{d}\right)=d \theta^{2}+\sinh ^{2} \theta d s^{2}\left(S^{d-1}\right)$ & $z=\frac{u}{\sqrt{1-u^{2}}+\cosh \theta}, z^{i}=\frac{\sinh \theta n^{i}}{\sqrt{1-u^{2}}+\cosh \theta}, n^{i} n^{i}=1$ \\
\hline
\end{tabular}

Table 9: The three slice metrics $d s_{d}^{2}$ corresponding respectively to $k=0, \pm 1$, and the coordinate transformations that bring the metric (7.25) with $\mu=0$ into the upper half plane metric (7.28).

The slice metric $d s_{d}^{2}$ is either one of the three constant curvature metrics given in table 9 or the metric on a quotient of these by a discrete subgroup of their isometry group. Moreover, since we picked the plus sign in the renormalized momentum $\widehat{\pi}_{\left(\Delta_{+}\right)}$in (4.6) so that the kinetic term in the effective action has a positive sign, we must pick the minus sign in (7.25).

Note that if either $k$ or $\mu$ are negative, then the range of the radial coordinate is bounded, $0 \leq u \leq u_{*}$, for some upper bound $u_{*} \cdot{ }^{23}$ For $k$ and $\mu$ non-negative, however, $u$ is unbounded from above: $0 \leq u<\infty$. Since $\phi=u^{(d-1) / 2} \varphi$, in this case regularity of the solution (7.25) requires that $\varphi \rightarrow 0$ as $u \rightarrow \infty .{ }^{24}$ This gives the condition

$$
\int_{\frac{d-1}{2 l \sqrt{\lambda}} \varphi_{o}^{-2 /(d-1)}}^{\infty} \frac{d v}{\sqrt{1+k v^{2}+\frac{d(d-1)}{\kappa^{2} l^{2} \lambda}\left(\frac{2 l \sqrt{\lambda}}{d-1}\right)^{d+1} \mu v^{d+1}}}=\int_{0}^{\infty} \frac{d v}{\sqrt{1+k v^{2}+\mu v^{d+1}}}
$$

For $\mu=0$ this is trivially satisfied since both integrals diverge. It follows that $\mu=0$ leads to regular solutions, which we will discuss shortly. For $\mu>0$, however, this constraint can only be satisfied if

$$
\lambda<\left(\frac{(d-1)^{d} \kappa^{2}}{2^{d+1} d l^{d-1}}\right)^{2 /(d-1)} .
$$

Provided this holds, the above constraint gives a relation between $\mu$ and the VEV $\phi_{-}$. Note, however, that for $d=2$ and $d=3$ the condition on the coupling is respectively $\lambda<\left(\kappa^{2} / 16 l\right)^{2}$ and $\lambda<\kappa^{2} / 6 l^{2}$. But recall that we have determined in (7.20) that for $d=2$ we must necessarily have $\lambda=\left(\kappa^{2} / 16 l\right)^{2}$, while for $d=3, \lambda=\kappa^{2} / 6 l^{2}$ is precisely the value of the coupling such that the action (7.3) can be embedded in M-theory. It follows that no regular solutions of the form (7.25) with both $k$ and $\mu$ non-negative exist in these two cases. This is particularly significant for the three-dimensional case that can be embedded in M-theory. Note that (7.25) with $k=1$ and $\mu>0$, were it a regular solution, it would

\footnotetext{
${ }^{23}$ In fact, for $k=-1$ and $\mu>0$ these solutions are very similar to the Janus solution [52].

${ }^{24}$ Note, however, that regularity of the solutions for the conformally coupled scalar $(7.3)$ does not guarantee the regularity of the corresponding minimally coupled scalar since the transformation rules (7.2) may break down. This should be checked case by case.
} 
be a gravitational instanton similar to the numerical solutions of [13]. As in that case, by analytic continuations (7.25) would then give static spherically symmetric gravitational solitons, as well as Big Bang/Crunch geometries, which would have a dual description in the dual $\mathcal{N}=8$ strongly coupled SCFT in three dimensions. Our argument shows the absence of such solitons in this theory. Of course, this refers to the particular truncation of $\mathcal{N}=8$ gauged supergravity that gives the action (7.3), and which is different from the one used in [13]. We will see in the next section, however, that (7.3) does admit regular instanton solutions, but of a different type.

An interesting property of the general domain wall solution (7.25) is that for $\mu=0$ the metric is the metric of Euclidean $\mathrm{AdS}_{d+1}$ (i.e. of the hyperbolic space $\mathbb{H}_{d+1}$ ), for all possible values of $k=0, \pm 1 .{ }^{25}$ Indeed, the coordinate transformations given in table 9 for each of the cases $k=0, \pm 1$, transform the metric in (7.25) with $\mu=0$ to the upper half plane metric of $\mathbb{H}_{d+1}$

$$
d s^{2}=\frac{l^{2}}{z^{2}}\left(d z^{2}+d z^{i} d z^{i}\right), \quad i=1, \ldots, d .
$$

Moreover, for $\mu=0$ the scalar field in (7.25) takes the form

$$
\phi=\varphi_{o} u^{\frac{d-1}{2}}\left(\sqrt{1+k u^{2}} \pm u \sqrt{k+\left(2 l \sqrt{\lambda} \varphi_{o}^{\frac{2}{d-1}} /(d-1)\right)^{2}}\right)^{-(d-1) / 2}
$$

where $\varphi_{o}$ is an arbitrary constant corresponding to the VEV $\phi_{-}$. For $k \geq 0, \varphi_{o} \geq 0$ but for $k=-1, \varphi_{o}>((d-1) l / \sqrt{2 \lambda})^{(d-1) / 2}$. Note also that with the sign choice we made above, the plus sign should be chosen in this expression. We will revisit this special case of the domain wall solutions (7.25) shortly.

Another special case of the general solution (7.25) deserves a comment. Namely, for $d=3$ and $\lambda=\kappa^{2} / 6 l^{2}$, in which case the two potentials (6.21) and (7.1) agree, the Poincaré domain wall (6.24) that we found in the previous section matches precisely with the domain wall (7.25) for $k=0$. In order to compare the two solutions we use the field redefinitions $(7.2)$ to rewrite the domain wall (6.24) in the frame where the scalar field is conformally coupled. The transformed solution takes the form

$$
\begin{aligned}
d s^{2} & =\frac{1}{4 \rho^{2}}\left(1+\nu \rho+\sqrt{1+2 \nu \rho+\rho^{2}}\right)\left(1+\sqrt{1-\rho^{2}}\right)\left(\frac{l^{2} d \rho^{2}}{\left(1-\rho^{2}\right)\left(1+2 \nu \rho+\rho^{2}\right)}+\rho_{o}^{2} d x^{i} d x^{i}\right), \\
\phi & =\frac{\sqrt{6}}{\kappa} \frac{\rho}{1+\sqrt{1-\rho^{2}}}
\end{aligned}
$$

where $\rho_{o}=\frac{2 \kappa}{\sqrt{6}} \phi_{-}$. It is now straightforward to check that identifying

$$
u^{-2}=\frac{\rho_{o}^{2}}{4 \rho^{2}}\left(1+\nu \rho+\sqrt{1+2 \nu \rho+\rho^{2}}\right)\left(1+\sqrt{1-\rho^{2}}\right),
$$

the solution (7.30) reproduces the domain wall (7.25), provided we also identify $\mu=\left(\nu^{2}-\right.$ 1) $\rho_{o}^{4} / 16$. According to the above discussion then, only the supersymmetric domain wall,

\footnotetext{
${ }^{25}$ Of course, this is true provided there are no global identifications in the slice metric $d s_{d}^{2}$.
} 
corresponding to $\nu=-1$, is regular. However, even in this case, the corresponding solution for the physically relevant minimally coupled scalar is not regular. As was discussed in [23], the difference between the supersymmetric and non-supersymmetric domain walls is that the former has a null singularity, while the later has a timelike singularity. In both cases this singularity can be seen in the corresponding 11-dimensional solution as arising from a collapsing $S^{2}$ inside the asymptotically $S^{7}$ transverse space.

\subsection{Instantons}

Finally, we consider a very special class of solutions of the equations of motion (7.4), which for the case $d=3$ were found in [24]. These are solutions that satisfy

$$
\mathcal{T}_{\mu \nu}=0, \quad R_{\mu \nu}+\frac{d}{l^{2}} g_{\mu \nu}=0 .
$$

In the case of a vanishing cosmological constant such solutions have been discussed in [53]. The vanishing of the modified stress tensor gives a linear equation for the scalar field, namely

$$
\left(\nabla_{\mu} \nabla_{\nu}-\frac{1}{d+1} g_{\mu \nu} \square_{g}\right) \phi^{-2 /(d-1)}=0,
$$

which admits non-trivial solutions provided the metric is exact $\mathrm{AdS}_{d+1}$. The general solution of this equation, subject to the constraint that it also satisfies the second equation in (7.4), can be written in the upper half plane coordinates (7.28) as

$$
\phi^{2 /(d-1)}=\frac{(d-1)}{l \sqrt{|\lambda|}}\left(\frac{b z}{-\operatorname{sgn}(\lambda) b^{2}+(z+a)^{2}+\left(\vec{z}-\vec{z}_{0}\right)^{2}}\right),
$$

where $a, b, z_{0}^{i}, i=1, \ldots, d$, are arbitrary constants. A special case of this solution (for $\lambda<0$ ) was found in [54] as a solution of the scalar equation (7.4) in four dimensions and ignoring the back-reaction on the geometry. It was later pointed out in 24] that for any value of the coupling, $\lambda$, there is in fact no back-reaction and, together with the $\mathrm{AdS}_{4}$ metric, this is an exact solution of the full gravity-scalar system. The solution (7.34) is the generalization of the exact solution of [24] to any dimension.

In order to understand the significance of the parameters in this solution we consider its asymptotic expansion

$$
\phi \sim e^{-\frac{(d-1) r}{2 l}} \phi_{-}(\vec{z})-l \alpha e^{-\frac{(d+1) r}{2 l}} \phi_{-}^{\frac{(d+1)}{(d-1)}}(\vec{z}),
$$

where $\alpha \equiv \sqrt{|\lambda|} a / b$ and the inhomogeneous $\operatorname{VEV}, \phi_{-}(\vec{z})$, is given by

$$
\phi_{-}^{2 /(d-1)}=\frac{(d-1)}{l \sqrt{|\lambda|}}\left(\frac{b}{-\operatorname{sgn}(\lambda) b^{2}+a^{2}+\left(\vec{z}-\vec{z}_{0}\right)^{2}}\right) .
$$

The asymptotic form (7.35) tells us that the two modes, $\phi_{ \pm}$, of this soliton are related by $\phi_{+}=-l \alpha \phi_{-}^{\frac{(d+1)}{(d-1)}}$, or equivalently

$$
\widehat{\pi}_{\left(\Delta_{+}\right)}=-\frac{1}{l}\left(\Delta_{+}-\Delta_{-}\right) \phi_{+}=\alpha \phi_{-}^{\frac{d+1}{2}} .
$$


From table 2 we then unambiguously conclude that the solution (7.34) satisfies Mixed boundary conditions: $J_{f_{-}}=-\widehat{\pi}_{\left(\Delta_{+}\right)}-f^{\prime}\left(\phi_{-}\right)=0$, with

$$
f\left(\phi_{-}\right)=-\alpha \frac{(d-1)}{2 d} \phi_{-}^{\frac{2 d}{(d-1)}}
$$

which corresponds to a marginal multi-trace deformation with deformation parameter $\alpha$. It follows that the parameter $\alpha=\sqrt{|\lambda|} a / b$ is not a modulus of the solution (i.e. of the VEV), but rather a modulus of the theory itself (of course this refers to the large $N$ limit only). Different values of $\alpha$ correspond to different points along the line of marginal deformations (7.38). Equivalently, two solutions of the form (7.34) with different values of $\alpha$ satisfy different boundary conditions. Note that regularity of the general solution (7.34) requires that $a>b \geq 0$ and hence $\alpha>\sqrt{\lambda}>0$. Using the exact effective potential (7.23) with the boundary condition (7.38) we see that this is precisely the condition for the effective potential to become unbounded from below. ${ }^{26}$ This suggests that the Euclidean solutions (7.34) are instantons which mediate the decay of the conformal vacuum at $\phi_{-}=0$ due to the instability introduced by the marginal deformation (7.38) [24].

A curious feature of the VEV (7.36) is that it is an extremum of a simple two-derivative boundary action [24]. ${ }^{27}$ This 'phenomenological' effective action in flat space takes the form

$$
S=\mathcal{C} \int d^{d} z\left(\phi_{-}^{-\frac{2}{d-1}} \partial^{i} \phi_{-} \partial_{i} \phi_{-}+\left(\lambda-\alpha^{2}\right) \phi_{-}^{\frac{2 d}{d-1}}\right)
$$

where $\mathcal{C}$ is an arbitrary constant. Note, however, that this effective action does not - and indeed it does not have to - agree with the holographic two-derivative effective action (7.11) we have derived above. If one chooses $\mathcal{C}$ such that it matches the correct coefficient of the kinetic term given in (7.11), then the coefficient of the potential term in (7.39) should be changed as $\lambda-\alpha^{2} \rightarrow 2 \sqrt{\lambda}(\sqrt{\lambda}-\alpha)$, if (7.39) were to agree with (7.11). Note that in the vicinity of the critical point at $\alpha=\sqrt{\lambda}$ these coefficients $d o$ actually agree. However, we see this as merely a curious coincidence, since the correct two-derivative effective action as we showed is (7.11), and indeed the instanton VEV (7.36), which is an exact extremum of the full all-derivative effective action, need not be an extremum of the two-derivative effective action.

Moduli space. The moduli space of the instantons (7.34) is the space parameterized by all arbitrary parameters of the solution, subject to the condition that the boundary conditions remain fixed, i.e. provided $\alpha$ remains fixed. The moduli space becomes manifest if we rewrite the instanton solution (7.34) in terms of the coordinates $\left(Y_{-1}, Y_{0}, Y_{i}\right), i=$ $1, \ldots, d$, of the covering space, $\mathbb{R}^{1, d+1}$, of $\mathbb{H}_{d+1}$. Namely, we introduce coordinates on

\footnotetext{
${ }^{26}$ Putting the theory on $S^{d}$ does not change this conclusion, as can be deduced from the effective potential 7.21 .

${ }^{27}$ See [55] for a recent discussion of the $d=2$ case.
} 
$\mathbb{R}^{1, d+1}$ as well as a set of auxiliary constants parameterizing an $\widetilde{\mathbb{H}}_{d+1}$ as

$$
\begin{array}{ll}
Y_{-1}=\frac{l}{2 z}\left(1+z^{2}+\vec{z}^{2}\right), & \tilde{Y}_{-1}=\frac{\tilde{l}}{2 \tilde{z}}\left(1+\tilde{z}^{2}+\vec{z}^{2}\right), \\
Y_{0}=\frac{l}{2 z}\left(1-z^{2}-\vec{z}^{2}\right), & \tilde{Y}_{0}=\frac{\tilde{l}}{2 \tilde{z}}\left(1-\tilde{z}^{2}-\tilde{\tilde{z}}^{2}\right), \\
Y_{i}=\frac{l}{z} z^{i}, & \tilde{Y}_{i}=\frac{\tilde{l}}{\tilde{z}} \tilde{z}^{i} .
\end{array}
$$

The solution (7.34) then can be written as

$$
\phi^{2 /(d-1)}=\frac{1}{-\tilde{Y} \cdot Y+\frac{2}{d-1} l \alpha},
$$

where $\tilde{Y} \cdot Y \equiv-\tilde{Y}_{-1} Y_{-1}+\tilde{Y}_{0} Y_{0}+\sum_{i=1}^{d} \tilde{Y}_{i} Y_{i}$, and we have identified

$$
\tilde{z}=\frac{(d-1)}{2} \frac{b \tilde{l}}{\sqrt{|\lambda|}}, \quad \tilde{z}^{i}=z_{o}^{i}, \quad \tilde{l}=\frac{2}{d-1} \sqrt{\alpha^{2}-\lambda} .
$$

The moduli space is therefore a hyperbolic space, $\tilde{\mathbb{H}}_{d+1}$, of radius $\tilde{l}$, which is well defined precisely for $\alpha>\sqrt{\lambda}$. Recall that this is exactly the condition for the instantons to exist, as well as, for the effective potential to be unbounded from below. Finally, note that the form (7.41) allows one to easily write the solution in any other coordinate system parameterizing $\mathbb{H}_{d+1}$.

Special limits. Since the metric (7.25) becomes exact $\operatorname{AdS}_{d+1}$ for $\mu=0$ and (7.34) is the most general solution corresponding to an exact $\mathrm{AdS}_{d+1}$ metric, it follows that we must be able to obtain the $\mu=0$ domain wall solutions discussed above as a limit of the solution (7.34). This can indeed be easily seen, provided we realize that in taking any limits of the general solution (7.34), we do not necessarily have to satisfy the the condition $a>b \geq 0$ that was essential for the regularity of the general solution. This is especially so since the special limits can satisfy more general boundary conditions than the general solution (7.34).

Setting first $a=b$ in (7.34) and letting $b \rightarrow \infty$ and $\vec{z}_{o}^{2} \rightarrow \infty$, while keeping $b / \vec{z}_{o}^{2}=$ $\frac{l \sqrt{\lambda}}{d-1} \varphi_{o}^{\frac{2}{d-1}}$ constant, reproduces the solution (7.29) with $k=0$. Moreover, setting $z_{o}^{i}=0$ and $b=\frac{2 l \sqrt{\lambda}}{d-1} \varphi_{o}^{\frac{2}{d-1}}$ in (7.34), the two choices $a= \pm \sqrt{ \pm 1+b^{2}}$ lead via the coordinate transformations given in table 9 to the solution (7.29) with $k= \pm 1$ respectively. It follows that, although all three solutions satisfy boundary conditions corresponding to the marginal deformation (7.38), only the solution for $k=1$ satisfies the condition $\alpha>\sqrt{\lambda}$, which is necessary in order for the solution to be identified with an instanton. For $k=0$ instead we have $\alpha=\sqrt{\lambda}$, while for $k=-1, \alpha<\sqrt{\lambda}$. This can be seen directly by looking at the extrema of the effective potential (7.21). Namely, the equation $V_{k}^{\prime}\left(\phi_{-}\right)=0$ is nothing but the gap equation $J_{f_{-}}=-\widehat{\pi}_{\left(\Delta_{+}\right)}-f^{\prime}\left(\phi_{-}\right)=0$, where now $\widehat{\pi}_{\left(\Delta_{+}\right)}$is given by (4.4) and $f\left(\phi_{-}\right)$ by (7.38). Rearranging this equation gives

$$
k+\left(\lambda-\alpha^{2}\right)\left(\frac{2 l}{d-1} \varphi_{o}^{\frac{2}{d-1}}\right)^{2}=0 .
$$




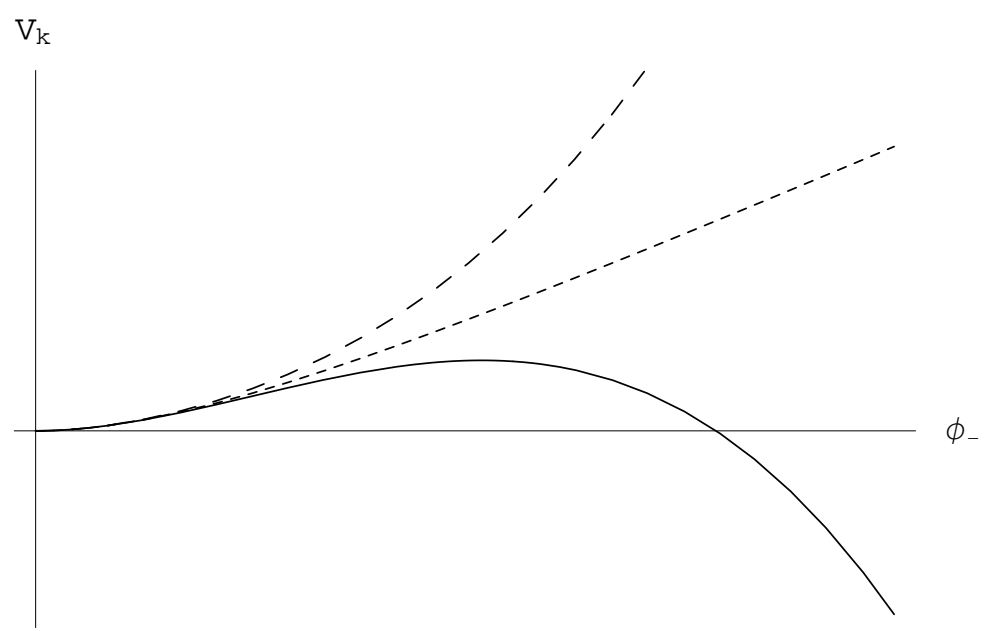

Figure 1: Plot of the effective potential (7.21), with $f\left(\phi_{-}\right)$given by (7.38), on $S^{3}$ for $\alpha<\sqrt{\lambda}$ (long dashes), $\alpha=\sqrt{\lambda}$ (short dashes), and $\alpha>\sqrt{\lambda}$.

Note that the VEV, $\phi_{o}$, is undetermined for $k=0$. In the special case where this solution is embedded into $\mathcal{N}=8$ gauged supergravity in four dimensions, this is precisely the Coulomb branch solution corresponding to $\nu=-1$ in (6.24). For $k=1$ the VEV is fixed to $\varphi_{o}^{\frac{2}{d-1}}=1 / l \tilde{l}$, which is a local maximum of the effective potential (7.21), as can be seen from figure 1 for the case $d=3$. This plot also shows that the effective potential on $S^{3}$ is stable, marginally stable and unstable according to whether $\alpha<\sqrt{\lambda}, \alpha=\sqrt{\lambda}$ and $\alpha>\sqrt{\lambda}$ respectively.

Vacuum decay rate. We have seen above that the existence of these instanton solutions for $\alpha>\sqrt{\lambda}$ coincides with the critical point where the exact quantum effective potential (7.21) becomes unbounded from below. This suggests that the dual field theory becomes unstable and the conformal vacuum at $\phi_{-}=0$ decays via quantum tunneling, mediated by these instantons, to something else [24]. The endpoint of this decay is unclear, however, since classical supergravity breaks down before this endpoint is reached. In the $d=3$ case, where the system $(7.3)$ can be embedded in $\mathcal{N}=8$ gauged supergravity and the corresponding asymptotically $A d S_{4} \times S^{7}$ solutions uplifted to 11-dimensional supergravity, this breakdown of the supergravity description can be traced to an $S^{2}$ inside the $S^{7}$ collapsing [24]. $1 / N$ corrections must therefore be taken into account in order to understand the endpoint of the decay. From the bulk point of view, the instantons signal an instability of the AdS vacuum once the modified boundary condition (7.38), with $\alpha>\sqrt{\lambda}$ is imposed.

The instanton solutions allow us to compute the decay probability of the conformal vacuum, which is given by [39]

$$
\left.\mathcal{P} \propto e^{-\Gamma_{\alpha}[\phi]}\right|_{\text {instanton }},
$$

where $\left.\Gamma_{\alpha}[\phi]\right|_{\text {instanton }}$ is the effective action of the boundary theory evaluated on the instanton VEV (7.36). Although we do not know the general form of the effective action, this 
decay rate can be computed exactly in two different ways. First, the effective action on the VEV (7.36) can be evaluated by computing the bulk on-shell action on the instanton solution (7.34), taking into account the boundary term (7.38). This gives

$$
\left.\Gamma_{\alpha}\right|_{\text {instanton }}=\frac{\pi^{\frac{d+1}{2}} \tilde{l}^{-d}}{\Gamma\left(\frac{d+1}{2}\right)} \frac{\alpha}{d}\left[1-\frac{d}{(d+1) \frac{\alpha}{\sqrt{\lambda}}\left(\frac{\alpha}{\sqrt{\lambda}}+1\right)} F\left(\frac{d+2}{2}, 1 ; d+2 ; \frac{2}{\frac{\alpha}{\sqrt{\lambda}}+1}\right)\right] .
$$

The second way relies on boundary quantities only. The crucial observation is that since the value of the on-shell action does not depend on the moduli of the instanton - indeed (7.45) depends only on the coupling $\lambda$ and the deformation parameter $\alpha$ - we can go to any point in the instanton moduli space to evaluate the effective action. In particular, we saw that there is a point in the moduli space where the instanton VEV (7.36) is constant, namely $\phi_{-}^{\frac{2}{d-1}}=1 / l \tilde{l}$. This corresponds to the $\mu=0, k=1$ domain wall we discussed above. But then the effective action reduces to the effective potential, which we have computed in (7.21). Evaluating the effective potential on this constant VEV and multiplying by the volume of $S^{d}$ gives, after some manipulation using the identity

$$
F\left(-\frac{d}{2}, \frac{1}{2} ; 1-\frac{d}{2} ;-x\right)=\sqrt{1+x}-\left(\frac{d-1}{d-2}\right) x F\left(1-\frac{d}{2}, \frac{1}{2} ; 2-\frac{d}{2} ;-x\right),
$$

precisely (7.45).

\section{Acknowledgments}

I would like to thank Jan de Boer, Sebastian de Haro, Anastasios C. Petkou, Kostas Skenderis and Jörg Teschner for very useful comments. I also thank Ecole Polytechnique for hospitality during the completion of this work.

\section{A. The variational problem and Hamilton-Jacobi equations}

In this appendix we consider the variational problem for the actions (6.1) and (7.3) on the regulating hypersurface $\Sigma_{r}$. Specifically, we determine the appropriate Gibbons-Hawking term and the radial canonical momenta for each case. Moreover, we give the radial Hamiltonian densities, which, via the Hamiltonian and momentum constraints, determine the corresponding Hamilton-Jacobi equations.

A generic variation of the bulk action produces a bulk term proportional to the equations of motion as well as a boundary term. Namely,

$$
\delta S=\int_{\mathcal{M}} d^{d+1} x \sqrt{g}\left((\mathrm{eoms})+\nabla_{\mu} v^{\mu}\right),
$$

for some vector field $v^{\mu}$. This vector field is fundamental to the study of the variational problem and the radial Hamiltonian formalism. For the actions (6.1) and (7.3) it is given respectively by

$$
\begin{aligned}
& v_{\text {min }}^{\mu}=-\frac{1}{\kappa^{2}} g^{\rho[\mu} \nabla^{\sigma]} \delta g_{\rho \sigma}+\delta \phi \nabla^{\mu} \phi \\
& v_{\text {conf }}^{\mu}=-\frac{1}{\kappa^{2}}\left(1-\frac{(d-1) \kappa^{2}}{4 d} \phi^{2}\right) g^{\rho[\mu} \nabla^{\sigma]} \delta g_{\rho \sigma}-\frac{d-1}{2 d} \phi \delta g_{\rho \sigma} g^{\rho[\mu} \nabla^{\sigma]} \phi+\delta \phi \nabla^{\mu} \phi .
\end{aligned}
$$




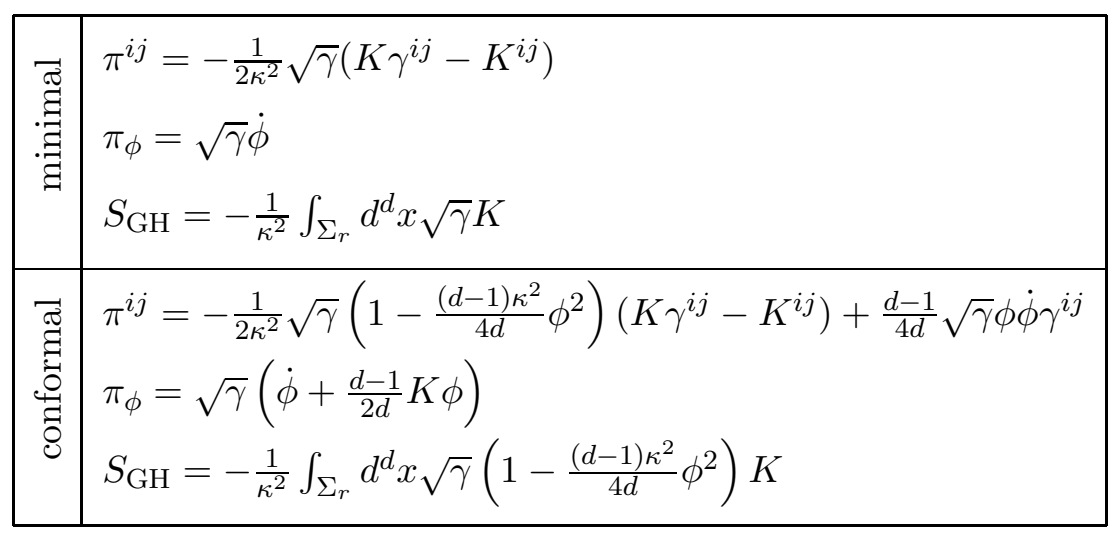

Table 10: The canonical radial momenta and the Gibbons-Hawking terms for the actions (6.1) and (7.3). Note that $K_{i j}=\frac{1}{2} \dot{\gamma}_{i j}$ is the extrinsic curvature of the hypersurface $\Sigma_{r}$.

We can evaluate explicitly the boundary term (A.1) on $\Sigma_{r}$ using the gauge-fixed metric (3.1). Generically it takes the form

$$
\mathcal{B}_{r} \equiv \int_{\Sigma_{r}} * v=-\delta S_{G H}+\int_{\Sigma_{r}} d^{d} x\left(\delta \gamma_{i j} \pi^{i j}+\delta \phi \pi_{\phi}\right),
$$

but the precise form of the Gibbons-Hawking term, $S_{G H}$, and of the radial canonical momenta, $\pi^{i j}$ and $\pi_{\phi}$, crucially depends on the form of the bulk action. In table 10 we give explicitly the momenta and the Gibbons-Hawking terms for minimally and conformally coupled scalars. Note that although the Gibbons-Hawking term for minimally coupled scalars is identical to the standard Gibbons-Hawking term for pure gravity, this is no longer true for conformally coupled scalars.

The radial momenta given in table 10 are, of course, the same quantities as those one would obtain from the functional derivatives of the off-shell bulk Lagrangian with respect to the radial derivative of the corresponding induced field, i.e.

$$
\pi^{i j}=\frac{\delta L}{\delta \dot{\gamma}_{i j}}, \quad \pi_{\phi}=\frac{\delta L}{\delta \dot{\phi}}
$$

However, the boundary term (A.4) shows that they also correspond to the functional derivatives of the the regularized on-shell action,

$$
\left.I_{r} \equiv\left(S+S_{\mathrm{GH}}\right)\right|_{\text {on-shell }},
$$

with respect to the induced fields on the hypersurface $\Sigma_{r}$. Namely,

$$
\pi^{i j}=\frac{\delta I_{r}}{\delta \gamma_{i j}}, \quad \pi_{\phi}=\frac{\delta I_{r}}{\delta \phi} .
$$

These relations, familiar from Hamilton-Jacobi theory, are the main reason why the radial Hamiltonian formalism is the most direct approach for studying the supergravity limit of the AdS/CFT correspondence. Indeed, in the simplest case of Dirichlet boundary conditions, the AdS/CFT dictionary identifies the induced fields, e.g. $\gamma_{i j}$ and $\phi$, with the sources 
of the dual operators and the regularized on-shell action with the generating functional of regularized connected correlation functions. It follows that the canonical momenta given by (A.7) correspond to the regularized one-point functions of the dual operators with arbitrary sources. This statement trivially carries over for renormalized correlation functions once the covariant boundary counterterms are added to $I_{r}$. Moreover, as it is extensively discussed in section 3, once the covariant boundary counterterms are added to $I_{r}$, one can add further appropriate finite boundary terms in order to modify the boundary conditions.

The bulk equations of motion can be written in terms of the radial canonical momenta using a 'radial ADM formalism'. As is well known, the resulting equations are the standard first order Hamilton equations complemented with the Hamiltonian and momentum constraints, which reflect the diffeomorphism invariance of the theory. For the actions (6.1) and (7.3) the constraints take the form

$$
\mathcal{H}=0, \quad 2 D_{i} \pi_{j}^{i}=\pi_{\phi} \partial_{j} \phi,
$$

where the Hamiltonian density, $\mathcal{H}$, is given respectively by

$$
\begin{aligned}
\mathcal{H}_{\text {min }}= & \frac{2 \kappa^{2}}{\sqrt{\gamma}}\left(\pi^{i j} \pi_{i j}-\frac{\pi^{2}}{d-1}\right)+\frac{1}{2 \sqrt{\gamma}} \pi_{\phi}^{2}-\sqrt{\gamma}\left(-\frac{1}{2 \kappa^{2}} R[\gamma]+\frac{1}{2} \partial^{i} \phi \partial_{i} \phi+V(\phi)\right), \\
\mathcal{H}_{\text {conf }}= & \frac{2 \kappa^{2}}{\sqrt{\gamma}}\left(1-\frac{(d-1) \kappa^{2}}{4 d} \phi^{2}\right)^{-1}\left(\pi^{i j} \pi_{i j}-\frac{1}{d} \pi^{2}\right)-\frac{2 \kappa^{2}}{d(d-1) \sqrt{\gamma}}\left(\pi-\frac{d-1}{4} \phi \pi_{\phi}\right)^{2} \\
& +\frac{1}{2 \sqrt{\gamma}} \pi_{\phi}^{2}-\sqrt{\gamma}\left(-\frac{1}{2 \kappa^{2}} R[\gamma]+\frac{(d-1)^{2}}{2 d(d-2)} \phi^{\frac{d}{d-1}} \Delta_{\gamma} \phi^{\frac{d-2}{d-1}}-\frac{d(d-1)}{2 \kappa^{2} l^{2}}+\frac{\lambda}{2} \phi^{\frac{2(d+1)}{(d-1)}}\right),
\end{aligned}
$$

and where

$$
\Delta_{\gamma} \equiv-\square_{\gamma}+\frac{(d-2)}{4(d-1)} R[\gamma]
$$

is the scalar conformal Laplacian in $d$ dimensions. Note that although the form of the Hamiltonian and of the momenta is different for minimally and conformally coupled scalars, the form of the constraints remains the same. Hamilton's equations can then be written in terms of the Hamiltonian $H=\int d^{d} x \mathcal{H}$ as

$$
\begin{aligned}
\dot{\gamma}_{i j} & =2 K_{i j}=\frac{\delta H}{\delta \pi^{i j}}, & \dot{\phi} & =\frac{\delta H}{\delta \pi_{\phi}}, \\
\dot{\pi}^{i j} & =-\frac{\delta H}{\delta \gamma_{i j}}, & \dot{\pi}_{\phi} & =-\frac{\delta H}{\delta \phi} .
\end{aligned}
$$

The two equations in the first line are just the inverse of the expressions in table 10 for the momenta in terms of the radial derivatives of the induced fields. The two equations in the second line give the second order equations one would obtain from the components of Einstein's equation that are transverse to $\Sigma_{r}$. However, we will not need the explicit form of these equations since we only use the Hamilton-Jacobi formalism in this paper. This consists in inserting the canonical momenta as derivatives of the regularized on-shell action (see (A.7) in the Hamilton and momentum constraints (A.8). The resulting equations are the Hamilton-Jacobi equations for the gravity-scalar system. Hamilton's equations are then automatically satisfied due to the identification (A.7). 


\section{References}

[1] E. Witten, Multi-trace operators, boundary conditions and AdS/CFT correspondence, hep-th/0112258.

[2] M. Berkooz, A. Sever and A. Shomer, Double-trace deformations, boundary conditions and spacetime singularities, JHEP 05 (2002) 034 hep-th/0112264.

[3] W. Muck, An improved correspondence formula for AdS/CFT with multi-trace operators, Phys. Lett. B 531 (2002) 301 [hep-th/0201100].

[4] P. Minces, Multi-trace operators and the generalized AdS/CFT prescription, Phys. Rev. D 68 (2003) 024027 hep-th/0201172.

[5] A. Sever and A. Shomer, A note on multi-trace deformations and AdS/CFT, JHEP 07 (2002) 027 hep-th/0203168.

[6] O. Aharony, M. Berkooz and B. Katz, Non-local effects of multi-trace deformations in the AdS/CFT correspondence, JHEP 10 (2005) 097 hep-th/0504177.

[7] S. Elitzur, A. Giveon, M. Porrati and E. Rabinovici, Multitrace deformations of vector and adjoint theories and their holographic duals, JHEP 02 (2006) 006 hep-th/0511061.

[8] S.S. Gubser and I.R. Klebanov, A universal result on central charges in the presence of double-trace deformations, Nucl. Phys. B 656 (2003) 23 hep-th/0212138.

[9] T. Hartman and L. Rastelli, Double-trace deformations, mixed boundary conditions and functional determinants in AdS/CFT, hep-th/0602106.

[10] D.E. Diaz and H. Dorn, Partition functions and double-trace deformations in AdS/CFT, hep-th/0702163.

[11] M. Henneaux, C. Martinez, R. Troncoso and J. Zanelli, Black holes and asymptotics of $2+1$ gravity coupled to a scalar field, Phys. Rev. D 65 (2002) 104007 hep-th/0201170.

[12] T. Hertog and K. Maeda, Black holes with scalar hair and asymptotics in $N=8$ supergravity, JHEP 07 (2004) 051 hep-th/0404261.

[13] T. Hertog and G.T. Horowitz, Towards a big crunch dual, JHEP 07 (2004) 073 hep-th/0406134.

[14] T. Hertog, An AdS crunch in supergravity, AIP Conf. Proc. 743 (2005) 305 hep-th/0409160.

[15] T. Hertog and K. Maeda, Stability and thermodynamics of AdS black holes with scalar hair, Phys. Rev. D 71 (2005) 024001 [hep-th/0409314].

[16] T. Hertog and G.T. Horowitz, Designer gravity and field theory effective potentials, Phys. Rev. Lett. 94 (2005) 221301 hep-th/0412169.

[17] T. Hertog and G.T. Horowitz, Holographic description of AdS cosmologies, JHEP 04 (2005) 005 hep-th/0503071.

[18] T. Hertog and S. Hollands, Stability in designer gravity, Class. and Quant. Grav. 22 (2005) 5323 hep-th/0508181.

[19] A.J. Amsel and D. Marolf, Energy bounds in designer gravity, Phys. Rev. D 74 (2006) 064006 [Erratum ibid. 75 (2007) 029901] hep-th/0605101. 
[20] T. Hertog, Violation of energy bounds in designer gravity, Class. and Quant. Grav. 24 (2007) 141 hep-th/0607171.

[21] A.J. Amsel, T. Hertog, S. Hollands and D. Marolf, A tale of two superpotentials: stability and instability in designer gravity, Phys. Rev. D 75 (2007) 084008 hep-th/0701038].

[22] P. Breitenlohner and D.Z. Freedman, Positive energy in anti-de Sitter backgrounds and gauged extended supergravity, Phys. Lett. B 115 (1982) 197.

[23] I. Papadimitriou, Non-supersymmetric membrane flows from fake supergravity and multi-trace deformations, JHEP 02 (2007) 008 [hep-th/0606038].

[24] S. de Haro, I. Papadimitriou and A.C. Petkou, Conformally coupled scalars, instantons and vacuum instability in $A d S_{4}$, hep-th/0611315.

[25] G.W. Gibbons, C.M. Hull and N.P. Warner, The stability of gauged supergravity, Nucl. Phys. B 218 (1983) 173.

[26] W. Boucher, Positive energy without supersymmetry, Nucl. Phys. B 242 (1984) 282.

[27] P.K. Townsend, Positive energy and the scalar potential in higher dimensional (super)gravity theories, Phys. Lett. B 148 (1984) 55.

[28] E. Witten, A simple proof of the positive energy theorem, Commun. Math. Phys. 80 (1981) 381 .

[29] J. de Boer, E.P. Verlinde and H.L. Verlinde, On the holographic renormalization group, JHEP 08 (2000) 003 hep-th/9912012.

[30] K. Skenderis, Lecture notes on holographic renormalization, Class. and Quant. Grav. 19 (2002) 5849 hep-th/0209067.

[31] I. Papadimitriou and K. Skenderis, Thermodynamics of asymptotically locally AdS spacetimes, JHEP 08 (2005) 004 hep-th/050519d.

[32] I. Papadimitriou and K. Skenderis, AdS/CFT correspondence and geometry, hep-th/0404176.

[33] S. de Haro, S.N. Solodukhin and K. Skenderis, Holographic reconstruction of spacetime and renormalization in the AdS/CFT correspondence, Commun. Math. Phys. 217 (2001) 595 hep-th/0002230.

[34] V. Balasubramanian, P. Kraus and A.E. Lawrence, Bulk vs. boundary dynamics in anti-de Sitter spacetime, Phys. Rev. D 59 (1999) 046003 hep-th/9805171.

[35] I.R. Klebanov and E. Witten, AdS/CFT correspondence and symmetry breaking, Nucl. Phys. B 556 (1999) 89 hep-th/9905104.

[36] P. Breitenlohner and D.Z. Freedman, Stability in gauged extended supergravity, Ann. Phys. (NY) $144(1982) 249$.

[37] I. Papadimitriou, Holographic renormalization made simple: an example, prepeared for International School of Subnuclear Physics, 41st Course: From Quarks to Black Holes: Progress in Understanding the Logic of Nature, Erice, Sicily, Italy, 29 Aug - 7 Sep (2003).

[38] I. Papadimitriou, Aspects of the gauge/gravity duality and holography, PhD Thesis, Universiteit van Amsterdam (2005), ISBN 9064642567, avaiable online at http://dare.uva.nl/document/15962. 
[39] S.R. Coleman and F. De Luccia, Gravitational effects on and of vacuum decay, Phys. Rev. D 21 (1980) 3305.

[40] I. Papadimitriou and K. Skenderis, Correlation functions in holographic RG flows, JHEP 10 (2004) 075 hep-th/0407071.

[41] J. Parry, D.S. Salopek and J.M. Stewart, Solving the Hamilton-Jacobi equation for general relativity, Phys. Rev. D 49 (1994) 2872 gr-qc/9310020].

[42] H. Goldstein, Classical mechanics, 2nd ed., Reading Addison-Wesley (1980).

[43] Dr. E. Kamke, Differentialgleichungen Lösungsmethoden und Lösungen, Chelsea Publishing Company (1971).

[44] M. Henningson and K. Skenderis, The holographic Weyl anomaly, JHEP 07 (1998) 023 hep-th/9806087.

[45] K. Skenderis and P.K. Townsend, Gravitational stability and renormalization-group flow, Phys. Lett. B 468 (1999) 46 hep-th/9909070.

[46] O. DeWolfe, D.Z. Freedman, S.S. Gubser and A. Karch, Modeling the fifth dimension with scalars and gravity, Phys. Rev. D 62 (2000) 046008 hep-th/9909134.

[47] V.L. Campos, G. Ferretti, H. Larsson, D. Martelli and B.E.W. Nilsson, A study of holographic renormalization group flows in $D=6$ and $D=3$, JHEP 06 (2000) 023 hep-th/0003151.

[48] D.Z. Freedman, C. Núñez, M. Schnabl and K. Skenderis, Fake supergravity and domain wall stability, Phys. Rev. D 69 (2004) 104027 hep-th/0312055.

[49] K. Skenderis and P.K. Townsend, Hamilton-Jacobi for domain walls and cosmologies, Phys. Rev. D 74 (2006) 125008 hep-th/0609056.

[50] M.J. Duff and J.T. Liu, Anti-de Sitter black holes in gauged $N=8$ supergravity, Nucl. Phys. B 554 (1999) 237 hep-th/9901149.

[51] C. Martinez, R. Troncoso and J. Zanelli, Exact black hole solution with a minimally coupled scalar field, Phys. Rev. D 70 (2004) 084035 hep-th/0406111.

[52] D. Bak, M. Gutperle and S. Hirano, A dilatonic deformation of $A d S_{5}$ and its field theory dual, JHEP 05 (2003) 072 hep-th/0304129.

[53] E. Ayon-Beato, C. Martinez, R. Troncoso and J. Zanelli, Gravitational cheshire effect: nonminimally coupled scalar fields may not curve spacetime, Phys. Rev. D 71 (2005) 104037 hep-th/0505086.

[54] S. de Haro and A.C. Petkou, Instantons and conformal holography, JHEP 12 (2006) 076 hep-th/0606276.

[55] A. Zamolodchikov and A. Zamolodchikov, Decay of metastable vacuum in Liouville gravity, hep-th/0608196. 